\title{
Pacific
}

Journal of

Mathematics

\section{A CLASS OF INCOMPLETE NON-POSITIVELY CURVED MANIFOLDS}

BRIAN BOWDITCH 


\title{
A CLASS OF INCOMPLETE NON-POSITIVELY CURVED MANIFOLDS
}

\author{
B.H. BowDITCH
}

In this paper, we describe a class of simply connected nonpositively curved riemannian manifolds which satisfy some curvature constraints. Such manifolds have many of the properties of (complete) Hadamard manifolds, such as geodesic convexity and the existence of an ideal boundary.

\section{Introduction.}

The geometry of Hadamard (complete, simply-connected, non-positively curved riemannian) manifolds has been intensively studied for some time. A general account of the basic theory can be found in [BaGS]. However, there are interesting examples of non-positively curved manifolds which fail to be complete, while retaining many of the geometric properties of Hadamard manifolds. The best known is the Weil-Peterssen metric on Teichmüller space. This is negatively curved [Ah, Tro] and incomplete [W1], yet it admits an exhaustion by compact convex sets, and is thus geodesically convex [W2]. We describe some further examples in Chapter 2. Also, incomplete non-positively curved metrics have been used to construct interesting examples of complete non-positively curved manifolds by modifying the metric in a neighbourhood of the ends (see for example [A $\mathbf{b S}]$ ).

These examples suggest that certain incomplete metrics may be of some interest in their own right. In this paper we restrict attention to metrics satisfying certain curvature constraints, and show that they behave, in many respects, like complete manifolds. We shall assume in particular that the curvature "blows up" along any path of finite length that leaves every compact set.

Let us first summarise a few properties of (complete) Hadamard manifolds. Firstly, the exponential map based at any point gives a diffeomorphism of $\mathbb{R}^{n}$ onto $X$. Moreover, there is a natural compactification, $X_{C}$, of $X$ into a topological ball, formed by adjoining the ideal sphere, $X_{I}=X_{C} \backslash X$. A point of $X_{I}$ may be thought of as an equivalence class of geodesic rays, where two rays are equivalent if they remain a bounded distance apart.

If, in addition, we assume that $X$ has strictly negative curvature bounded away from 0 , then it follows that $X$ is a "visibility manifold", i.e. any two points of $X_{I}$ may be joined by a bi-infinite geodesic [EO]. 
If we go further, and impose another curvature bound away from $-\infty$ (so that $X$ has "pinched curvature"), then much more can be said about the geometry of $X$. For example, we have Anderson's result [An] that if $Q \subseteq X_{C}$ is any closed subset, and hull $(Q) \subseteq X_{C}$ is the closed convex hull of $Q$, then $X_{I} \cap \operatorname{hull}(Q)=X_{I} \cap Q$. For further results about convex sets, see [Bo].

To generalise to incomplete (i.e. not necessarily complete) manifolds, let us assume that:

(A) $X$ is a Riemannian manifold such that

(A1) $X$ has non-positive curvature, and

(A2) $X$ is simply connected.

We write $d$ for the path-metric on $X$, and write $(\bar{X}, d)$ for the metric completion of $(X, d)$. Given $x \in X$, write $\kappa(x)$ for the maximal sectional curvature of any tangent 2-plane at $X$.

Suppose we assume, in addition to (A), that:

(B) For all $a \in \bar{X} \backslash X$, there is some $K>0$ and a neighbourhood $U$ of $a$ in $\bar{X}$ such that for all $x \in X \cap U$, we have $\kappa(x) \leq-1 / K^{2} d(x, a)^{2}$;

then, we claim that:

(1) $X$ is geodesically convex. In fact, any two points $x, y \in \bar{X}$ may be joined by a geodesic segment $[x, y] \subseteq X \cup\{x, y\}$. Moreover, $[x, y]$ is, up to reparameterisation, uniquely length-minimising among all rectifiable paths in $\bar{X}$.

(2) The completion $\bar{X}$ is a CAT(0) space (as explained in Section 3.5).

(3) There is a natural compactification $X_{C}$ of $X$ so that $X_{C}$ is homeomorphic to a closed ball, with $X$ as its interior.

(4) There is a natural continuous injection $\iota: \bar{X} \longrightarrow X_{C}$ from $\bar{X}$ in the metric topology to $X_{C}$ in its topology as a ball.

(5) Suppose $(x, y) \in\left(X_{C} \times X_{C}\right) \backslash\left(X_{I}^{\infty} \times X_{I}^{\infty}\right)$ where $X_{I}^{\infty}=X_{C} \backslash \iota(\bar{X})$. Then, $x$ and $y$ may be joined by a unique geodesic $[x, y] \subseteq X \cup\{x, y\}$, (where $[x, x]=\{x\}$ ). Moreover, $[x, y]$ is closed in $X_{C}$.

(6) The map $[(x, y) \mapsto[x, y]]:\left(X_{C} \times X_{C}\right) \backslash\left(X_{I}^{\infty} \times X_{I}^{\infty}\right) \longrightarrow \mathscr{C}\left(X_{C}\right)$ is continuous, where $\mathscr{C}\left(X_{C}\right)$ is the set of all closed subsets of $X_{C}$ in the Hausdorff topology (Section 5.2).

Suppose, in addition to (A) and (B), that $X$ satisfies:

(C) There exist $p_{0} \in X$ and $L_{0}, R_{0}>0$, such that if $x \in X$ with $d\left(x, p_{0}\right) \geq$ $R_{0}$, then $\kappa(x) \leq-1 / L_{0}^{2} d\left(x, p_{0}\right)^{2}$; then it follows also that:

$\left(5^{\prime}\right)$ If $(x, y) \in X_{C} \times X_{C}$, then $x$ and $y$ may be joined by a unique geodesic $[x, y] \subseteq X \cup\{x, y\}$.

$\left(6^{\prime}\right)$ The map $[(x, y) \mapsto[x, y]]: X_{C} \times X_{C} \longrightarrow \mathscr{C}\left(X_{C}\right)$ is continuous. 
More precise statements of these results will be given later. They will all be proven in this paper: (1) Proposition 3.5.3. (2) Proposition 3.5.1, (3) Proposition 4.5.2, (4) Proposition 4.3.4, (5) Lemma 4.1.4, Lemma 5.3.1, (6) Proposition 5.3.4, (5') Lemma 6.2.1, Proposition 6.2.3, (6 $\left.6^{\prime}\right)$ Proposition 6.3.2.

If one adds additional hypotheses, such as pointwise pinching of curvature, then we have variations of Anderson's construction which enable us to construct convex sets in $X$. Thus, for example, with appropriate hypotheses, we can deduce that $X$ has an exhaustion by compact convex sets. There is also the possibility of generalising some of the results of [Bo] to such spaces, though we shall not get involved with that here. Indeed we suspect that this programme could be carried further, and that, for example, many analytic results could be carried over to such spaces.

Note that in the complete case, pinched negative curvature is the same as pointwise negative curvature together with bounded geometry. "Bounded geometry" means that, for any fixed $r>0$, the set of metric balls $\{N(x, r) \mid$ $x \in X\}$ (defined up to isometry) all lie in a compact set in the $C^{2}$-topology. There is an analogous statement in the incomplete case. In this case, if $X$ is negatively curved, properties (B) and (C) and pointwise pinching of curvature are all implied by a single hypothesis of "bounded geometry up to scale". To explain what we mean, let $B$ be the closed unit ball in $\mathbb{R}^{n}$, with a standard orthonormal frame, $F_{0}$, at the origin, $o$. Let $\mathscr{S}$ be the space of smooth Riemannian metrics on $B$, with strictly negative curvature and with smooth boundary, $\partial B$, such that the frame $F_{0}$ is orthonormal in each metric, and such that $\partial B$ is always the unit sphere about $o$. We give the space $\mathscr{S}$ the $C^{2}$ topology. Suppose that $X$ satisfies (A). Suppose that $x \in X$, and $\lambda>0$ is such that the ball $N(x, \lambda)$ is compact. Given any orthonormal frame, $F$, at $x$, let $e: B \longrightarrow N(x, \lambda)$ be the composition of a dilation by a factor of $\lambda$ on $\mathbb{R}^{n}$ with the exponential map sending $F_{0}$ to $F$. Thus, $e$ is a diffeomorphism, so we can pull back the metric on $X$ to get a metric on $B$. This gives us a point of $\mathscr{S}$. We shall say that $X$ has bounded geometry up to scale if there is a compact subset, $S \subseteq \mathscr{S}$, such that for all $x \in X$, we can choose $\lambda(x)>0$ such that $N(x, \lambda(x))$ is compact, and such that for some frame at $x$, the the point of $\mathscr{S}$ constructed as above always lies in $S$. (Note that we are free to choose $\lambda(x)$ as small as we like. However, the sectional curvatures at the origin of metrics in $S$ are all bounded away from 0 . Thus, if $\lambda(x)$ is small, the scaling factor forces the curvature at $x$ to be large. Similarly, if the curvature at $x$ is small, then there must be a large compact metric ball centred on $x$.) We leave as an exercise the fact that this property implies properties (B) and (C).

As remarked earlier, one motive for studying incomplete manifold might 
be to gain some further insight into the geometry of the Weil-Peterssen metric on the Teichmüller spaces. Wolpert [W2] shows that this is geodesically convex. As an example, he considers the case of once-punctured tori. In this case, the moduli space is a 2-dimensional Riemannian orbifold with two cone singularities (orbifold points), and a cusp singularity (with the cusp point removed), of the type obtained by spinning the graph of $f(x)=x^{3}, x>0$ about the $x$-axis. It follows that the universal cover (i.e. Teichmüller space) in this case satisfies axioms (A) and (B) (see Chapter 2). For higher-dimensional spaces, the situation becomes more complicated. The asymptotics of the curvature tensor have been studied by Trapani [Tra]. It appears that in general property (B) fails. However, one might still hope for some modification of the hypothesis (B), for example, to take account to the directions of the tangent 2-planes along which the curvature blows up, sufficient to recover an ideal sphere analogous to Thurston's compactification.

In general, incomplete simply connected manifolds of negative curvature seem to have received little attention. Without some strong constraints on the curvature, they can behave in ways quite unlike Hadamard manifolds. For example, Hass [Ha] gives an example of a negatively curved metric on a 3 -ball which contains a closed geodesic in its interior. This phenomenon is not possible in dimension 2, nor with constant curvature in any dimension. It might be interesting to explore further conditions under which this sort of behaviour would be prohibited.

\section{Examples.}

In this chapter we give some examples of the kind of incomplete manifolds we are considering. These particular examples have been chosen principally to illustrate the assertions made in the introduction. We begin with some manifolds satisfying properties (A) and (B).

Suppose $-\infty \leq a<b \leq \infty$, and that $f:(a, b) \longrightarrow(0, \infty)$ is a smooth function. Let $t$ be an arc-length parameter along the graph of $f, \operatorname{graph}(f) \subseteq$ $(a, b) \times(0, \infty)$. Given $t \in \operatorname{graph}(f)$ write $\rho(t) \in \mathbb{R} \cup\{\infty\}$ for the length of the tangent at this point to the intercept with the $x$-axis. (Figure 2.) We take the sign of $\rho(t)$ to be the same as that of $d f / d t$. We may form a surface of revolution, $S$, by spinning graph $(f)$ about the $x$-axis. Now, $S$ has two orthogonal foliations: one by generators of $S$ which are intrinsically geodesic, and the other by circles of curvature $c(t)=1 / \rho(t)$. We see that $S$ has Gaussian curvature equal to

$$
k(t)=-\frac{d c}{d t}-c^{2}=\frac{1}{\rho^{2}}\left(\frac{d \rho}{d t}-1\right)=-\frac{1}{f} \frac{d^{2} f}{d t^{2}} .
$$

Thus, for $S$ to non-positively curved, we need that $f$ be convex. Such a surface, $S$, has two topological ends corresponding to the ends of the interval 
$(a, b)$. We see that the end corresponding to $a$ will be complete if and only if $a=-\infty$, or else $a>-\infty$ and $f(x) \rightarrow \infty$ as $x \rightarrow a$. We call such an end a tube. If we have $a>-\infty$ and $f(x) \rightarrow 0$ and $\frac{d f}{d x}(x) \rightarrow 0$ as $x \rightarrow a$, then we call the end a cusp. If $S$ satisfies property (B), we see that it is necessary (but not sufficient) that either both the ends of $S$ be tubes, or that one end be a tube, and the other be a cusp.

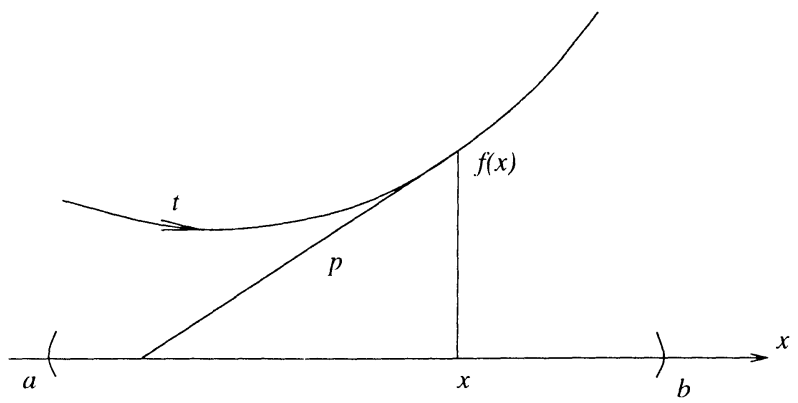

Figure 2.

As an explicit example, consider the graph of $f(x)=x^{\beta}$ for some $\beta>1$, defined on the interval $(0, \infty)$. We have $-\frac{1}{f(x)} \frac{d^{2} f}{d x^{2}}(x)=-\beta(\beta-1) x^{-2}$. Now $x / t \rightarrow 1$ as $t \rightarrow 0$, and so the curvature of $S$ blows up like $-1 / t^{2}$ as we approach the cusp point at 0 . We see that $S$ satisfies (A1) and (B), and so its universal cover, $X=\tilde{S}$ satisfies (A) and (B). The metric completion $\bar{X}$ of $X$ is obtained by adding a single point, $p$, at the origin 0 . Thus, under the natural inclusion $\iota: \bar{X} \rightarrow X_{C}$, the point $p$ maps to an ideal point $\iota(p) \in X_{I}$. The remaining ideal points can be thought of as the endpoints of the geodes: generators of $X$, as $t \rightarrow \infty$. Thus, the set $X_{I}^{\infty}$ of these remaining ideal points has naturally the topology of an open interval. This is compactified into the circle, $X_{I}$, by adding the point $\iota(p)$.

Suppose, more generally, that $f:(0, b) \longrightarrow(0, \infty)$ is convex, and that $f(x) \rightarrow 0$ and $\frac{d f}{d x}(x) \rightarrow 0$ as $x \rightarrow 0$. Then $\mu=\lim _{t \rightarrow \infty} \frac{d f}{d t}(t) \in(0,1]$ is well defined. (Thus $\mu=1$ if $b \leq \infty$.) Let $S$ be the surface of revolution, and $X=\tilde{S}$ the universal cover. We may coordinatise $X$ using a radial coordinate $\theta \in \mathbb{R}$ and an arc length coordinate $t \in(0, \infty)$. In this way, $S$ is the quotient of $X$ by the map $[(t, \theta) \mapsto(t, \theta+2 \pi)]$. As before, $X_{C}$ is formed by adjoining the arc $\{(\infty, \theta) \mid \theta \in \mathbb{R}\}$, and then taking the one-point compactification with the point 0 at the origin. Let $l_{\theta}$ be the geodesic generator $\{(t, \theta) \mid t \in(0, \infty)\}$ of $X$. The total Gauss curvature of the sector of $X$ lying between $l_{\theta_{1}}$ and $l_{\theta_{2}}$ may be calculated as $C\left(\theta_{0}\right)=-\int_{0}^{\infty}\left(\theta_{0} f\right)\left(\frac{1}{f} \frac{d^{2} f}{d t^{2}}\right) d t=-\mu \theta_{0}$ where $\theta_{0}=$ $\theta_{2}-\theta_{1}$. Applying Gauss-Bonnet, we find that the ideal points $\left(\infty, \theta_{1}\right)$ and $\left(\infty, \theta_{2}\right)$ can be joined by a bi-infinite geodesic in $X$ if and only if $C\left(\theta_{0}\right)<-\pi$ 
i.e. if and only if $\theta_{0}>\pi / \mu$. Now, $\mu \leq 1$, and so $X$ cannot have the visibility property. Note that $\frac{d \rho}{d t} \rightarrow 1$ as $t \rightarrow \infty$, and so $k(t)=\frac{1}{\rho^{2}}\left(\frac{d \rho}{d t}-1\right)=o\left(1 / t^{2}\right)$. Thus Property (C) fails in this case.

By giving similar consideration to the case where both ends of $S$ are tubes, we see that no surface constructed in this way can satisfy all of properties (A), (B) and (C).

The surfaces of revolution just described are a special case of the following more general construction.

Suppose $M$ is a Riemannian manifold, and that $I \subseteq \mathbb{R}$ is an open interval. Let $f: I \longrightarrow(0, \infty)$ be a smooth function. We define a Riemannian metric on $X=M \times I$ by setting

$$
d s^{2}=d t^{2}+f(t)^{2} \sum_{i, j} g_{i j} d x^{i} d x^{j}
$$

where $t$ is arc length in $I, g_{i j}$ is the Riemannian metric on $M$ with respect to the local coordinate system $\left(x^{i}\right)_{i}$, and $d s$ is infinitesimal distance in $X$.

We remark that this is an example of a still more general construction of "warped products" described in the paper of Bishop and O'Neill [BiO]. In a warped product, the interval $I$ may be replaced by any non-positively curved manifold. In the paper cited, there is a complete characterisation of when a warped product is non-positively curved.

In our special case, we can derive the relevant inequalities fairly simply as follows. Note that $X$ has two orthogonal foliations, one by geodesics of the form $\{x\} \times I$ for $x \in M$, and the other by codimension-1 submanifolds of the form $M_{t}=M \times\{t\}$ for $t \in I$. Each $M_{t}$ is totally umbilic, with principal curvatures equal to $c(t)=\frac{1}{f(t)} \frac{d f}{d t}(t)$. In the intrinsic metric, $M_{t}$ is isometric to $M$ with the metric scaled by a factor of $f(t)$.

Write $A=\partial / \partial t$ for the vector field on $X$ orthogonal to the $M_{t}$. Now suppose that $\Pi$ is a tangent 2-plane at $(x, t) \in M \times I=X$. If $\Pi$ is orthogonal to $A(x, t)$, then $\Pi$ corresponds to a tangent 2-plane, $\Pi_{M}$ at $x$ in $M$. Write $S_{M}\left(\Pi_{M}\right)$ for the sectional curvature of $M$ in $\Pi_{M}$. Thus, the sectional curvature, in $\Pi$, of the intrinsic metric of $M_{t}$ is $S_{M}\left(\Pi_{M}\right) / f(t)^{2}$. Applying Gauss's Theorema Egregium [S], we see that the sectional curvature, $S(\Pi)$, of $X$ in $\Pi$ is given by

$$
S(\Pi)=\frac{1}{f^{2}} S_{M}\left(\Pi_{M}\right)-c^{2}=\frac{1}{f^{2}}\left(S_{M}\left(\Pi_{M}\right)-\left(\frac{d f}{d t}\right)^{2}\right) .
$$

On the other hand, suppose that $\Pi$ is a tangent 2-plane at $(x, t)$ containing the vector $A(x, t)$. In this case the sectional curvature, $S(\Pi)$, of $X$ in $\Pi$ is

$$
S(\Pi)=-\frac{d c}{d t}-c^{2}=-\frac{1}{f} \frac{d^{2} f}{d t^{2}} .
$$


Now, if $Y$ and $Z$ are, locally, any two vector fields everywhere orthogonal to $A$, then a simple calculation shows that $R(A, Y, Y, Z)=0$, where $R$ is the Riemann curvature tensor. This symmetry implies that each sectional curvature of $X$ at $(x, t)$ lies between $-\frac{1}{f} \frac{d^{2} f}{d t^{2}}$ and $\frac{1}{f^{2}} S_{M}\left(\Pi_{M}\right)-c^{2}=$ $\frac{1}{f^{2}}\left(S_{M}\left(\Pi_{M}\right)-\left(\frac{d f}{d t}\right)^{2}\right)$ for some tangent 2-plane $\Pi_{M}$ at $x$ in $M$. In particular, for $X$ to be non-positively curved, it is sufficient that $M$ be nonpositively curved, and that $f$ be convex. (For more detailed computations of this nature, see [BiO].)

Examples of this construction are the surfaces of revolution described above. In this case, we have $M=\mathbb{R}$ and $f$ is thought of as a function of arc-length, $t$, along $\operatorname{graph}(f) \equiv I$. In such a case, we must always have $\frac{d f}{d t}<1$.

With this last constraint removed, we can construct examples satisfying (A), (B) and (C). For example, with $M=I=\mathbb{R}$, and $f(t)=e^{t}$, we obtain the hyperbolic plane foliated by horospheres.

For another example, set $M=\mathbb{R}, I=(0, \infty)$ and $f(t)=t^{\beta}$ with $\beta>$ 1. Now, the curvature $k(t)$ equals $-\frac{1}{f} \frac{d^{2} f}{d t^{2}}=-\beta(\beta-1) / t^{2}$. This case is qualitatively similar to the surface of revolution of $\left[x \mapsto x^{\beta}\right]$ described above, except that now, $X$ satisfies $(C)$, and has the visibility property.

As a third example, set $M=\mathbb{R}, I=(0,1)$ and $f(t)=t^{2} /(1-t)^{2}$. We see that $k(t)=-\frac{1}{f} \frac{d^{2} f}{d t^{2}}=-2(2 t+1) / t^{2}(1-t)^{2}$. Thus $-k(t)$ grows like $1 / t^{2}$ as $t \rightarrow 0$ and like $1 /(1-t)^{2}$ as $t \rightarrow 1$. It follows that $X$ satisfies (A) and (B). Since it is bounded (has finite diameter), it trivially satisfies (C). Both the completion, $\bar{X}$, and the compactification, $X_{C}$, of $X$ may be identified set-theoretically as $(\mathbb{R} \times[0,1]) / \sim$, where $(x, 0) \sim(y, 0)$ for all $x, y \in \mathbb{R}$. However, the topologies are different. Thus $X_{C}$ may be thought of as the one point compactification of $X \times(0,1]$ by adding the point $0 \equiv\{(x, 0)\} / \sim$, whereas $\bar{X}$ is noncompact-a base of neighbourhoods of 0 being given by $\{(\mathbb{R} \times[0, \epsilon)) / \sim \mid \epsilon>0\}$. Note that the natural map $\bar{X} \longrightarrow X_{C}$ is a continuous bijection.

One can construct higher dimensional examples, for example by taking $M$ to be euclidean $n$-space $\mathbb{E}^{n}$, or hyperbolic $n$-space $\mathbb{H}^{n}$. Note that $M=\mathbb{E}^{n}$, $I=\mathbb{R}$ and $f(t)=e^{t}$ gives us $\mathbb{H}^{n+1}$. So does $M=\mathbb{H}^{n}, I=\mathbb{R}$ and $f(t)=$ $\cosh t$.

There are many variations on this theme one can explore. One can also go on to construct further examples by gluing together examples of this type. 


\section{Geodesic convexity.}

In this chapter, we aim at establishing properties (1) and (2) for manifolds satisfying (A) and (B). The following notation is used throughout.

Suppose $X$ is a Riemannian manifold. We write $T_{x} X$ for the tangent space to $X$ at $x$, and $T X$ for the total space of the tangent bundle. Given $\xi, \zeta \in$ $T_{x} X$, we write $\langle\xi, \zeta\rangle$ and $|\xi|=\sqrt{\langle\xi, \xi\rangle}$ respectively for the Riemannian inner product and norm on $T_{x} X$. If $\xi, \zeta \neq 0$, set $\angle(\xi, \zeta)=\cos ^{-1}(\langle\xi, \zeta\rangle /|\xi \| \zeta|) \in$ $[0, \pi]$ for the angle between $\xi$ and $\zeta$. We write $d$ for the induced path-metric on $X$.

We shall use the term "geodesic" in the Riemannian sense of a curve whose first derivative is parallel. Thus, in terms of the metric $d$, a geodesic can be characterised as a constant-speed path, for which all sufficiently small subpaths are length-minimising.

3.1. Ruled maps. In this section we take $X$ to be a Riemannian manifold of non-positive curvature (A1). For $x \in X$, we write $\kappa(x) \in[-\infty, 0]$ to be the maximal sectional curvature at $x$.

Suppose that $I=\left[t_{0}, t_{1}\right] \subseteq \mathbb{R}$ is a closed interval and $J \subseteq \mathbb{R}$ is any interval. We write int $I$ and int $J$ respectively for the interiors of $I$ and $J$. Given a smooth map $\beta: I \times J \longrightarrow X$, we shall denote by $\beta_{u}$ and $\beta^{t}$ the maps

$$
\beta_{u}=[t \mapsto \beta(t, u)]: I \longrightarrow X
$$

and

$$
\beta^{t}=[u \mapsto \beta(t, u)]: J \longrightarrow X
$$

where $t \in I$ and $u \in J$. Thus $\beta_{u}(t)=\beta^{t}(u)=\beta(t, u)$. We refer to paths of the form $\beta_{u}$ and $\beta^{t}$ respectively as longitudes and transversals. We write $\partial \beta / \partial t$ and $\partial \beta / \partial u$ respectively for $\beta_{*}(\partial / \partial t)$ and $\beta_{*}(\partial / \partial u)$. We say that $\beta$ is a ruled map if for all $u \in I, \beta_{u}$ is a geodesic. Thus $\left|\frac{\partial \beta}{\partial t}(t, u)\right|=\left(\right.$ length $\left.\beta_{u}\right) / \mid t_{1}-$ $t_{0} \mid$.

Suppose that for $u \in J$, the geodesic $\beta_{u}$ is non-constant. We see that the $\operatorname{map}\left[t \mapsto \frac{\partial \beta}{\partial u}(t, u)\right]$ is the first variation of a geodesic along $\beta_{u}$. Thus, the component of $\frac{\partial \beta}{\partial u}(t, u)$ parallel to $\frac{\partial \beta}{\partial t}(t, u)$ is linear in $t$. Moreover, since $X$ is non-positively curved, the Riemannian norm of the component orthogonal to $\frac{\partial \beta}{\partial t}(t, u)$ is convex (see the discussion of normalised ruled maps below). It follows that the map $\left[t \mapsto\left|\frac{\partial \beta}{\partial u}(t, u)\right|\right]$ is convex. This is also readily verified in the case where $\beta_{u}$ is constant. Integrating, we find that the map $[t \mapsto$ length $\left.\beta^{t}\right]: J \longrightarrow[0, \infty)$ is convex. In particular:

Lemma 3.1.1. For all $t \in\left[t_{0}, t_{1}\right]$, we have

$$
\text { length } \beta^{t} \leq \max \left(\text { length } \beta^{t_{0}} \text {, length } \beta^{t_{1}}\right) \text {. }
$$


We shall say that a ruled map $\beta: I \times J \longrightarrow X$ is non-degenerate if $\beta_{u}$ is non-constant for all $u \in J$. In such a case, we say that $(t, u) \in I \times J$ is a singular point if $\beta$ fails to be an immersion at that point, i.e. if $\frac{\partial \beta}{\partial u}(t, u)$ is some multiple of $\frac{\partial \beta}{\partial t}(t, u)$. We say that $\beta$ is non-singular if there are no singular points in int $I \times J$. In such a case, the pull back of the Riemannian metric to int $I \times$ int $J$ is also a Riemannian metric of non-positive curvature. In fact, the curvature at $(t, u)$ is at most $\kappa(\beta(t, u))$. This is Synge's Inequality (see [S]). In the particular context of ruled maps, it is discussed in a paper of Aleksandrov [Alek].

By a ruled surface, we shall mean the image, $P=\beta(I \times J) \subseteq X$, of a ruled map $\beta: I \times J \longrightarrow X$, where $J$ is compact, and such that $\beta$ is nonsingular and injective on $\operatorname{int} I \times \operatorname{int} J$. We shall refer to the sets $\beta(I \times\{u\})$ for $u \in J$ as generating geodesics. We write $\kappa_{P}(x)$ for the intrinsic curvature of $P$ at $x$. Thus $\kappa_{P}(x) \leq \kappa(x) \leq 0$. Of particular interest is the case where the boundary, $\partial P$, of $P$ is a piecewise geodesic path. This motivates the following definition.

Definition. By a (non-positively curved) $n$-gon we mean a surface $P$, which is topologically a closed disc with boundary $\partial P$, together with a set $V \subseteq \partial P$ of $n$ points, and a metric, $\rho$ on $P$ such that $\rho$ restricted to the interior int $P=P \backslash \partial P$ is a non-positively curved Riemannian metric, and such that each component of $\partial P \backslash V$ is geodesic.

We shall refer to the points of $V$ as vertices and the components of $\partial P \backslash V$ as edges. At each vertex $v \in V$, the adjacent edges meet at some well-defined angle $\theta(v) \geq 0$. Since the metric is not assumed to be Riemannian at the point $v$ itself, it may be possible to have $\theta(v)=0$ (if the curvature grows sufficiently fast as we approach $v$ ). In such a case, we refer to $v$ as a cusp. In all cases we consider, $P$ will be convex, i.e. $\theta \leq \pi$ for all $v \in V$. Now, the Gauss-Bonnet formula tells us that

$$
\sum_{v \in V} \theta(v)=(n-2) \pi+\int_{P} \kappa_{P}(x) d \omega(x)
$$

where $\kappa_{P}(x)$ is the curvature at $x \in P$, and $d \omega$ is the area element. Note that we must always have $n \geq 3$.

By talking about ruled surfaces, we avoid having to worry about the technical complication of dealing with singular points; although intuitively. we would expect such points to work in our favour since they concentrate negative curvature. The fact that singular points do not cause any real problems has been made precise by Aleksandrov [Alek].

Another another type of restriction we shall want to place on ruled maps is the following. 
We say that a non-degenerate ruled map $\beta: I \times J \longrightarrow X$ is normalised if: (R1) for all $u \in J$, the longitude $\beta_{u}=[t \mapsto \beta(t, u)]$ is a geodesic parameterised with respect to arc-length (i.e. $\left|\frac{\partial \beta}{\partial t}(t, u)\right|=1$ for all $(t, u)$ ); and

(R2) for all $(t, u) \in I \times J$, we have

$$
\left\langle\frac{\partial \beta}{\partial t}(t, u), \frac{\partial \beta}{\partial u}(t, u)\right\rangle=0 .
$$

Thus, for a fixed $u$, the map $\left[t \mapsto \frac{\partial \beta}{\partial u}(t, u)\right]$ is a Jacobi field along the longitude $\beta_{u}$. We write $J(t)=\left|\frac{\partial \beta}{\partial u}(t, u)\right|$. From the Jacobi field equation $[\mathbf{S}]$, we know that, except where it vanishes, $J(t)$ is smooth in $t$, and that

$$
\frac{d^{2} J}{d t^{2}}(t) \geq-\kappa(\beta(t, u)) J(t)
$$

Suppose that $\lambda: I \longrightarrow[0, \infty)$ satisfies $\lambda(t) \leq-\kappa(\beta(t, u))$ for all $t \in I=$ $\left[t_{0}, t_{1}\right]$. The following is a simple consequence of the above differential inequality.

Proposition 3.1.2. Suppose $f: I \longrightarrow[0, \infty)$ is smooth and satisfies $\frac{d^{2} f}{d t^{2}}(t)=\lambda(t) f(t)$ for all $t \in I$. If $f\left(t_{0}\right)=J\left(t_{0}\right)$ and $\frac{d f}{d t}\left(t_{0}\right) \leq \frac{d J}{d t}\left(t_{0}\right)$ then $f(t) \leq J(t)$ for all $t \in I$.

Corollary 3.1.3. Suppose $f: I \longrightarrow[0, \infty)$ is smooth and satisfies $\frac{d^{2} f}{d t^{2}}(t)=$ $\lambda(t) f(t)$ for all $t \in I$. If $f\left(t_{0}\right)=J\left(t_{0}\right)$ and $f\left(t_{1}\right)=J\left(t_{1}\right)$, then $f(t) \leq J(t)$ for all $t \in I$.

Of particular interest will be the case where $\lambda$ has the form

$$
\lambda(t)=1 / K^{2}(t+h)^{2}
$$

for $t \geq 0$, and $K, h>0$ fixed. The solutions of $\frac{d^{2} f}{d t^{2}}(t)=\lambda(t) f(t)$ have the form $(t+h)^{1+\mu}$ and $(t+h)^{-\mu}$ where $\mu=\left(\sqrt{1+4 K^{2}}\right)-1>0$. In particular, if $f(0)=1$ and $\frac{d f}{d t}(0)=0$ we have the solution

$$
f(t)=\frac{\mu}{2 \mu+1}\left(\left(1+\frac{t}{h}\right)^{1+\mu}+\left(1+\frac{1}{\mu}\right)\left(1+\frac{t}{h}\right)^{-\mu}\right) .
$$

We shall refer to this later (Lemmas 3.4.1 and 6.1.1).

For the proof Lemma 3.4.1, we will need to describe a process of "normalising" ruled maps.

Suppose that $\alpha: I \times J \longrightarrow X$ is a non-degenerate ruled map, where now $I=\left[v_{0}, v_{1}\right]$. We are looking for a subset $S \subseteq \mathbb{R} \times J$ and a map $\rho: I \times J \longrightarrow S$ with the following properties:

(N1) $\rho$ is a smooth diffeomorphism of $I \times J$ onto $S$. 
(N2) For all $u \in J$, the set $S \cap(\mathbb{R} \times\{u\})$ is a closed interval of the form $\left[q_{0}(u), q_{1}(u)\right] \times\{u\}$.

(N3) For all $u \in J$, the map $\rho \mid(I \times\{u\})$ sends $I \times\{u\}$ linearly onto $\left[q_{0}(u), q_{1}(u)\right] \times\{u\}$.

(N4) The map $\beta=\alpha \circ \rho^{-1}: S \longrightarrow X$ is a normalised ruled map (i.e. it satisfies properties (R1) and (R2) above.)

We see that $S$ has the form $S=\left\{(t, u) \in \mathbb{R} \times J \mid q_{0}(u) \leq t \leq q_{1}(u)\right\}$, where $q_{0}, q_{1}: J \longrightarrow \mathbb{R}$ are smooth maps.

As before, we define longitudes, $\alpha_{u}, \beta_{u}$, and transversals $\alpha^{v}, \beta^{t}$, by $\alpha^{v}(u)=$ $\alpha_{u}(v)=\alpha(v, u)$ and $\beta^{t}(u)=\beta_{u}(t)=\beta(t, u)$. For $i=0,1$, set $\gamma_{i}=\alpha^{v_{i}}$ : $J \longrightarrow X$, and $\sigma_{i}=\left[u \mapsto\left(q_{i}(u), u\right)\right]: J \longrightarrow S$. Thus, $\gamma_{i}=\beta \circ \sigma_{i}$. We see that

$$
\frac{d \gamma_{i}}{d u}(u)=\frac{d q_{i}}{d u}(u) \frac{\partial \beta}{\partial t}\left(\sigma_{i}(u)\right)+\frac{\partial \beta}{\partial u}\left(\sigma_{i}(u)\right)
$$

and so

$$
\frac{d q_{i}}{d u}(u)=\left\langle\frac{d \gamma_{i}}{d u}(u), \frac{\partial \beta}{\partial t}\left(\sigma_{i}(u)\right)\right\rangle .
$$

Note that $\frac{\partial \beta}{\partial t}\left(\sigma_{i}(u)\right)$ is the unit tangent vector $\xi_{i}(u)=\left(\frac{v_{1}-v_{0}}{l(u)}\right) \frac{\partial \alpha}{\partial v}\left(v_{i}, u\right)$ to the geodesic $\alpha_{u}$, where $l(u)=$ length $\alpha_{u}=$ length $\beta_{u}$.

Now, suppose that we are given $\alpha$, and want to construct $S$ and $\rho$, and hence $\beta$. We can obtain the functions $q_{i}$, up to an additive constant, by integrating the quantity $\left\langle\frac{d \gamma_{i}}{d u}(u), \xi_{i}(u)\right\rangle$. Note that $\frac{d}{d u}\left(q_{1}(u)-q_{0}(u)\right)=\frac{d l}{d u}(u)$, and so we can arrange that $q_{1}(u)-q_{0}(u)=l(u)$ for all $u \in J$. This, then, defines the set $S \subseteq \mathbb{R} \times J$, and hence determines the map $\rho: I \times J \longrightarrow S$. One verifies that the map $\beta=\alpha \circ \rho^{-1}$ satisfies properties (R1) and (R2) as required.

3.2. The space of geodesics. For the moment, we can take $X$ to be any Riemannian manifold. Let $(\bar{X}, d)$ be the metric completion of $(X, d)$. Since $(X, d)$ is a path-metric space it follows that $(\bar{X}, d)$ is a path-metric space. We claim that every point of $\bar{X} \backslash X$ is accessible by a smooth path of finite length:

Lemma 3.2.1. Suppose $y \in \bar{X} \backslash X$; then there is a smooth path $\beta:[0,1] \longrightarrow$ $\bar{X}$ so that $\beta(0)=y, \beta((0,1]) \subseteq X$ and length $\beta<\infty$.

Proof. Certainly, $y$ is accessible by a rectifiable path of finite length in $X$, and we may use local convexity to approximate it by a smooth path.

Now, write $\operatorname{path}(\bar{X})$ for the set of all paths from $[0,1]$ to $\bar{X}$. Given $\alpha, \beta \in$ $\operatorname{path}(\bar{X})$, write

$$
d_{\text {sup }}(\alpha, \beta)=\max \{d(\alpha(t), \beta(t)) \mid t \in[0,1]\} .
$$


Thus $d_{\text {sup }}$ is a metric on $\operatorname{path}(\bar{X})$. We see easily that:

Proposition 3.2.2. $\left(\operatorname{path}(\bar{X}), d_{\text {sup }}\right)$ is a complete metric space. $X$.

We write $\operatorname{path}(X) \subseteq \operatorname{path}(\bar{X})$ for the subspace of paths lying entirely in We define the endpoint map

$$
\pi: \operatorname{path}(\bar{X}) \longrightarrow \bar{X} \times \bar{X}
$$

by $\pi(\beta)=(\beta(0), \beta(1))$. Clearly $\pi$ is continuous.

Let $\operatorname{geod}(\bar{X}) \subseteq \operatorname{path}(\bar{X})$ be the subspace of those $\beta \in \operatorname{path}(\bar{X})$ such that either $\beta$ is constant, or else $\beta((0,1)) \subseteq X$ and $\beta \mid(0,1)$ is a constant-speed geodesic. Let

$$
\operatorname{geod}(X)=\operatorname{geod}(\bar{X}) \cap \operatorname{path}(X)=\operatorname{geod}(\bar{X}) \cap \pi^{-1}(X \times X) .
$$

Now, let us suppose that $X$ is non-positively curved (A1). In this case, the $\operatorname{map} \pi: \operatorname{geod}(X) \longrightarrow X \times X$ is a local homeomorphism:

Lemma 3.2.3. Suppose $b \in \operatorname{geod}(X)$. Let $\pi(\beta)=(x, y)$. Then, there are neighbourhoods $U$ of $x$ and $V$ of $y$ in $X$, and a neighbourhood $W$ of $\beta$ in $\operatorname{geod}(X)$ such that $\pi \mid W: W \longrightarrow U \times V$ is a homeomorphism.

Proof. This follows, exactly as in the complete case, using the Jacobi field equation, and the implicit function theorem.

We see that, if $X$ has dimension $n$, then $\operatorname{geod}(X)$ is a $2 n$-dimensional manifold, and inherits a smooth structure from $X \times X$.

Suppose that $\gamma: J \longrightarrow \operatorname{geod}(X)$ is a smooth path. By definition, the paths $\gamma_{i}=[u \mapsto \gamma(u)(i)]: J \longrightarrow X$ for $i=0,1$ are smooth. We write $\hat{\gamma}:[0,1] \times J \longrightarrow X$ for the map given by $\hat{\gamma}(t, u)=\gamma(u)(t)$.

Lemma 3.2.4. The map $\hat{\gamma}$ is smooth.

Proof. From the implicit function theorem, exactly as in the complete case.

Thus, $\hat{\gamma}$ is a ruled map. Note that $\gamma_{\imath}=\hat{\gamma}^{i}$ according to our previous notation. Applying Lemma 3.1.1, we see that $\gamma$ is a rectifiable path in $\left(\operatorname{path}(\bar{X}), d_{\text {sup }}\right)$. In fact, if $J^{\prime} \subseteq J$ is any subinterval, then

$$
\text { length }\left(\gamma \mid J^{\prime}\right) \leq \max \left(\text { length }\left(\gamma_{0} \mid J^{\prime}\right), \text { length }\left(\gamma_{1} \mid J^{\prime}\right)\right) .
$$

Since (path $(\bar{X}), d_{\text {sup }}$ ) is complete, we have the following: 
Lemma 3.2.5. Suppose $\gamma:(0,1] \longrightarrow \operatorname{geod}(X)$ is smooth, and length $\gamma_{i}<$ $\infty$ for $i=0,1$. Then, $\gamma$ extends (uniquely) to a map $\gamma:[0,1] \longrightarrow \operatorname{path}(\bar{X})$.

Suppose, in such a case, it happens that $\gamma(0)((0,1)) \subseteq X$, so that $\gamma(0) \mid(0,1)$ must be geodesic. Thus, by definition, $\gamma(0) \in \operatorname{geod}(\bar{X})$. Our aim in the next section is to show that this is always the case if $X$ satisfies axiom (B), and $\gamma(0)$ is non-constant.

3.3. The path-lifting property. Suppose that $X$ is non-positively curved (A1) and satisfies:

(B) For all $a \in \bar{X} \backslash X$, there is some $K>0$ and a neighbourhood $U$ of $a$ in $\bar{X}$ such that for all $x \in X \cap U$ we have $\kappa(x) \leq-1 / K^{2} d(x, a)^{2}$.

We aim to show that $\pi: \operatorname{geod}(X) \longrightarrow X \times X$ is a covering map. A similar idea can be found in [AlexB]. This result will be based on the following path-lifting property.

Lemma 3.3.1. Suppose $\gamma:[0,1] \longrightarrow \operatorname{path}(\bar{X})$ with $\gamma((0,1]) \subseteq \operatorname{geod}(X)$, and $\gamma \mid(0,1]$ smooth. For $i=0,1$, write $\gamma_{i}$ for the path $[u \mapsto \gamma(u)(i)]$ : $[0,1] \longrightarrow \bar{X}$. Suppose that for $i=0,1$, we have length $\gamma_{i}<\infty$. Then $\gamma(0) \in \operatorname{geod}(\bar{X})$.

Proof. By definition, any constant path lies in $\operatorname{geod}(\bar{X})$, so we can suppose that $\gamma(0)$ is non-constant. As remarked at the end of the last section, it suffices to show that $\gamma(0)((0,1)) \subseteq X$. Without loss of generality, we can suppose that $\gamma(u)$ is non-constant for all $u \in[0,1]$. Define $\alpha:[0,1]^{2} \rightarrow \bar{X}$ by $\alpha(v, u)=\gamma(u)(v)$. Thus, $\alpha:[0,1] \times(0,1]$ is a non-degenerate ruled map. Now, the normalising procedure of Section 3.1 gives us a map $\rho$ : $[0,1] \times(0,1] \longrightarrow \mathbb{R} \times(0,1]$ so that $\beta=\alpha \circ \rho^{-1}: S_{0} \longrightarrow X$ is a normalised ruled map, where $S_{0}=\rho([0,1] \times(0,1])=\left\{(t, u) \in \mathbb{R} \times(0,1] \mid q_{0}(u) \leq t \leq q_{1}(u)\right\}$. We have $\gamma_{\imath} \mid(0,1]=\beta \circ \sigma_{i}$ where $\sigma_{i}(u)=\left(q_{i}(u), u\right)$. Thus, for all $u \in(0,1]$,

$$
\frac{d \gamma_{i}}{d u}(u)=\frac{d q_{i}}{d t}(u) \frac{\partial \beta}{\partial t}\left(\sigma_{i}(u)\right)+\frac{\partial \beta}{\partial u}\left(\sigma_{i}(u)\right)
$$

and so

$$
\left|\frac{d q_{\imath}}{d t}(u)\right| \leq\left|\frac{d \gamma_{i}}{d u}(u)\right|
$$

We see that $\int_{0}^{1}\left|\frac{d q_{2}}{d t}(u)\right| d u \leq$ length $\gamma_{i}<\infty$, and so $q_{i}(u)$ tends to a limit, $q_{i}(0)$, as $u$ tends to 0 . Also, since $l(u)=$ length $\alpha_{u}=q_{1}(u)-q_{0}(u)$ for all $u \in(0,1]$, and since $\alpha_{0}=\gamma(0)$ is non-constant, we see that $q_{0}(0)<$ $q_{1}(0)$. Let $S=\left\{(t, u) \in \mathbb{R} \times[0,1] \mid q_{0}(u) \leq t \leq q_{1}(u)\right\}$. We may extend $\rho$ to a homeomorphism $\rho:[0,1]^{2} \longrightarrow S$ mapping $[0,1] \times\{0\}$ linearly to $\left[q_{0}(0), q_{1}(0)\right] \times\{0\}$. Thus, $\beta$ extends to a map $\beta=\alpha \circ \rho^{-1}: S \longrightarrow \bar{X}$. As 
before, we define longitudes, $\beta_{u}$, and transversals, $\beta^{t}$, by $\beta_{u}(t)=\beta^{t}(u)=$ $\beta(t, u)$. We want to show that $\beta_{0}\left(\left(q_{0}, q_{1}\right)\right)=\gamma(0)((0,1)) \subseteq X$.

Suppose, for contradiction, that there is some $t \in\left(q_{0}, q_{1}\right)$ with $\beta(t, 0) \in$ $\bar{X} \backslash X$. For notational convenience, we shall assume that $t=0$, i.e. that $\beta(0,0) \in \bar{X} \backslash X$. Let $a=\beta(0,0)$.

Let $U$ be the neighbourhood of $a$ in $\bar{X}$ given by the hypothesis (B) above. We can find $t_{0}>0$ and $u_{0}>0$ such that $\left[-t_{0}, t_{0}\right] \times\left[0, u_{0}\right] \subseteq S$ and $\beta\left(\left[-t_{0}, t_{0}\right] \times\right.$ $\left.\left[0, u_{0}\right]\right) \subseteq U$.

Now, for all $(t, u) \in S$, we have that

$$
\left|\frac{\partial \beta}{\partial u}(t, u)\right| \leq \max _{i=0,1}\left|\frac{\partial \beta}{\partial u}\left(\sigma_{1}(u)\right)\right| \leq \max _{i=0,1}\left|\frac{d \gamma_{i}}{d u}(u)\right| .
$$

The first inequality follows from Corollary 3.1.3 (with $\lambda \equiv 0$ ) and the second comes from the formula for $\frac{d \gamma_{i}}{d u}(u)$ given above. In particular, we see that for all $t \in\left[-t_{0}, t_{0}\right]$,

$$
\int_{0}^{u_{0}}\left|\frac{\partial \beta}{\partial u}(t, u)\right| d u \leq \max _{i=0,1} \operatorname{length}\left(\gamma_{i} \mid\left[0, u_{0}\right]\right)<\infty .
$$

Given $u \in\left[0, u_{0}\right]$, set

$$
h(u)=\int_{0}^{u}\left|\frac{\partial \beta}{\partial u}(0, w)\right| d w .
$$

Thus, $h(u)=\operatorname{length}\left(\beta^{0} \mid[0, u]\right) \geq d(a, \beta(0, u))$. Since the longitude $\beta_{u}$ is a geodesic parameterised by arc-length, we have, for all $t \in\left[-t_{0}, t_{0}\right]$

$$
d(\beta(0, u), \beta(t, u))=|t|
$$

and so

$$
d(a, \beta(t, u)) \leq|t|+h(u) .
$$

Thus, by hypothesis (B), we have

$$
\kappa(\beta(t, u)) \leq-1 / K^{2}(|t|+h(u))^{2} .
$$

Fix, for the moment, some $u \in\left(0, u_{0}\right]$. For $t \in\left[-t_{0}, t_{1}\right]$ set $J(t)=$ $\left|\frac{\partial \beta}{\partial u}(t, u)\right|$. If $J(0) \neq 0$, then $J$ is differentiable at 0 . Suppose $\frac{d J}{d t}(0) \geq 0$. Then, applying Proposition 3.1 .2 on the interval $\left[0, t_{0}\right]$ and using the formula given after the Proposition, we find that

$$
J\left(t_{0}\right) \geq \frac{\mu}{2 \mu+1}\left(1+\frac{t_{0}}{h(u)}\right)^{1+\mu} J(0) .
$$


If, on the other hand, $\frac{d J}{d t}(0) \leq 0$, then, by symmetry, we get the same lower bound for $J\left(-t_{0}\right)$. Thus, in all cases, we get that

$$
J\left(-t_{0}\right)+J\left(t_{0}\right) \geq \frac{\mu}{2 \mu+1}\left(1+\frac{t_{0}}{h(u)}\right)^{1+\mu} J(0) .
$$

Thus,

$$
\begin{aligned}
\infty & >\int_{0}^{u_{0}}\left|\frac{\partial \beta}{\partial u}\left(-t_{0}, u\right)\right| d u+\int_{0}^{u_{0}}\left|\frac{\partial \beta}{\partial u}\left(t_{0}, u\right)\right| d u \\
& \geq \frac{\mu}{2 \mu+1} \int_{0}^{u_{0}}\left(1+\frac{t_{0}}{h(u)}\right)^{1+\mu}\left|\frac{\partial \beta}{\partial u}(0, u)\right| d u \\
& =\frac{\mu}{2 \mu+1} \int_{0}^{u_{0}}\left(1+\frac{t_{0}}{h(u)}\right)^{1+\mu} \frac{d h}{d u}(u) d u \\
& =\frac{\mu}{2 \mu+1} \int_{0}^{h\left(u_{0}\right)}\left(1+\frac{t_{0}}{w}\right)^{1+\mu} d w \\
& =\infty
\end{aligned}
$$

This contradicts the existence of $a \in \gamma(0)((0,1)) \cap(\bar{X} \backslash X)$. Thus $\gamma(0)((0,1)) \subseteq$ $X$, and so $\gamma(0) \in \operatorname{geod}(\bar{X})$ as required.

Corollary 3.3.2. The map $\pi: \operatorname{geod}(X) \longrightarrow X \times X$ is a covering map.

Proof. By Lemma 3.2.3, we know that $\pi$ is a local homeomorphism. Lemmas 3.2.5 and 3.3.1 together tell us that $\pi$ has the path-lifting property for smooth paths. The result follows by standard arguments.

3.4. Properties of geodesics. In this section we shall add the assumption (A2) that $X$ is simply connected, i.e., altogether we are assuming that $X$ satisfies hypotheses (A) and (B).

Now, $X \times X$ is simply connected, and so by Corollary 3.3 .2 , we see that each component of geod $(X)$ maps homeomorphically to $X \times X$ under $\pi$. Choose any point $x_{0} \in X$, and let $\operatorname{geod}_{0}(X)$ be the component of $\operatorname{geod}(X)$ containing the constant path at $x_{0}$. Let $\pi_{0}$ be the restriction of $\pi$ to $\operatorname{geod}_{0}(X)$ so that $\pi_{0}: \operatorname{geod}_{0}(X) \longrightarrow X \times X$ is a homeomorphism. Given $x, y \in X$, write $[x \rightarrow y]=\pi_{0}^{-1}(x, y)$. We see easily that for all $x \in X,[x \rightarrow x]$ is the constant path at $x$.

Lemma 3.4.1. $\operatorname{geod}(X)=\operatorname{geod}_{0}(X)$.

Proof. Suppose, for contradiction, that $\operatorname{geod}(X) \neq \operatorname{geod}_{0}(X)$. Choose any $x \in X$. Since $\pi: \operatorname{geod}(X) \longrightarrow X \times X$ is a covering map, there is some 
$\alpha \in \operatorname{geod}(X) \backslash \operatorname{geod}_{0}(X)$ with $\pi(\alpha)=(x, x)$. Thus $\alpha \neq[x \rightarrow x]$. Without loss of generality can suppose that $x \notin \alpha((0,1))$. (Otherwise choose a smaller segment of $\alpha$ and reparameterise.) For each $t \in(0,1)$, the path $\alpha$ meets the path $[x \rightarrow \alpha(t)]$ in $\alpha(t)$, at an angle different from 0 or $\pi$. Thus, as $t$ ranges through $[0,1]$, the geodesics $[x \rightarrow \alpha(t)]$ span a non-positively curved 1-gon, which is impossible by Gauss-Bonnet (Section 3.1).

In summary, we have shown:

Proposition 3.4.2. Any two points of $X$ are joined by a unique geodesic (defined on the domain $[0,1]$ ). Moreover, this geodesic varies smoothly in its endpoints.

Given $x, y \in X$, write $[x, y] \subseteq X$ for the image of $[x \rightarrow y]$. Thus $[x, x]=$ $\{x\}$ and $[x, y]=[y, x]$.

For a fixed $x \in X$, the function $\rho$ defined by $\rho(z)=\operatorname{length}[x \rightarrow z]$ is smooth on $X \backslash\{x\}$. Moreover, any geodesic $[x \rightarrow y]$ is orthogonal to the level sets of $\rho$, and so a standard argument of Riemannian geometry shows that:

Proposition 3.4.3. For all $x, y \in X$, the geodesic $[x \rightarrow y]$ is, up to reparameterisation, the unique length-minimising rectifiable path from $x$ to $y$. (In particular, $d(x, y)=$ length $[x \rightarrow y]$.)

Now, given $x \in X$ and $y \in X \backslash\{x\}$, we write $\overrightarrow{x y}=\frac{1}{d(x, y)} \frac{d \alpha}{d t}(0)$, where $\alpha=[x \rightarrow y]$. In other words, $\overrightarrow{x y}$ is the unit tangent vector at $x$ along $[x, y]$. If $z \in X \backslash\{x\}$, write $y \hat{x} z=\angle(\vec{x}, \vec{x} z)$ for the angle between $\overrightarrow{x y}$ and $\overrightarrow{x z}$.

Given the existence and uniqueness of geodesics, the following comparison theorems follow exactly as in the complete case. Let $\left(\mathbb{E}^{2}, d^{\prime}\right)$ be the euclidean plane,

Proposition 3.4.4. (Angle Comparison Theorem of Aleksandrov). Suppose $x, y, z \in X$ are distinct points. Choose $x^{\prime}, y^{\prime}, z^{\prime} \in \mathbb{E}^{2}$, so that $d^{\prime}\left(x^{\prime}, y^{\prime}\right)=$ $d(x, y), d^{\prime}\left(y^{\prime}, z^{\prime}\right)=d(y, z)$ and $d^{\prime}\left(z^{\prime}, x^{\prime}\right)=d(z, x)$. Then $x \hat{y} z \leq x^{\prime} \hat{y}^{\prime} z^{\prime}$, $y \hat{z} x \leq y^{\prime} \hat{z}^{\prime} x^{\prime}$ and $z \hat{x} y \leq z^{\prime} \hat{x}^{\prime} y^{\prime}$.

We refer to $x^{\prime} y^{\prime} z^{\prime}$ as a comparison triangle for $x y z$.

Proposition 3.4.5. (CAT $(0)$ inequality). Suppose $x, y, z \in X$ are distinct points. Suppose $u \in[x, y]$ and $v \in[x, z]$. Choose a comparison triangle. $x^{\prime} y^{\prime} z^{\prime}$ in $\mathbb{E}^{2}$ for $x y z$. Let $u^{\prime} \in\left[x^{\prime}, y^{\prime}\right]$ and $v^{\prime} \in\left[x^{\prime}, z^{\prime}\right]$ be the points with $d^{\prime}\left(x^{\prime}, u^{\prime}\right)=d(x, u)$ and $d^{\prime}\left(x^{\prime}, v^{\prime}\right)=d(x, v)$. Then $d^{\prime}\left(u^{\prime}, v^{\prime}\right) \leq d(u, v)$.

We thus say that $(X, d)$ is a "CAT(0)-space". More precisely, a $\operatorname{CAT}(0)$ space is a path-metric space in which every pair of points may be joined 
by a "geodesic", in the sense of a length-minimising path, and where the conclusion of Proposition 3.4.5 is satisfied where $[x, y]$ may be interpreted as any choice of geodesic from $x$ to $y$. In fact, it follows, in retrospect, that in a CAT(0)-space, there is a unique geodesic joining any pair of points, and so $[x, y]$ is uniquely defined. For further discussion of such spaces, see Ballmann's article in Chapter 10 of $[\mathbf{G H}]$, or the book by Bridson and Haefliger [BrH].

As a corollary of Proposition 3.4.5, we have the convexity of the distance function:

Proposition 3.4.6. Suppose $I, J \subseteq \mathbb{R}$ are intervals, and that $\alpha: I \longrightarrow X$ and $\beta: J \longrightarrow X$ are geodesics parameterised proportionately to arc-length. Then the function $[(t, u) \mapsto d(\alpha(t), \beta(u))]: I \times J \longrightarrow[0, \infty)$ is convex.

3.5. The completion. Finally in this chapter, we describe the geometry of the completion $(\bar{X}, d)$ of $(X, d)$. We are again assuming that $X$ satisfies hypotheses (A) and (B).

Now, the metric completion of any $\operatorname{CAT}(0)$-space is a $\operatorname{CAT}(0)$-space, so we see immediately that:

Proposition 3.5.1. $(\bar{X}, d)$ is a $C A T(0)$-space.

In particular, every pair of points are joined by a unique geodesic. Recall, however, that the term "geodesic" is here being used in the metric space sense of a constant-speed globally length-minimising path. We should therefore check that this agrees with the notion of "geodesic" already defined in Section 3.2. As before, we write $\operatorname{geod}(\bar{X})$ for the space of such geodesics.

Note that it's easy to see that a path $\alpha \in \operatorname{geod}(\bar{X})$ is globally lengthminimising, in other words, that length $\alpha=d(x, y)$ where $(x, y)=\pi(\alpha)$. To do this, choose $t \in\left(0, \frac{1}{2}\right]$. Since geodesics in $X$ are globally length-minimising (Proposition 3.4.3), we have that length $(\alpha \mid(t, 1-t))=d(\alpha(t), \alpha(1-t))$. The observation follows by letting $t \rightarrow 0$. Now, since $(\bar{X}, d)$ is $\operatorname{CAT}(0)$, it now follows that if $\alpha, \beta \in \operatorname{geod}(\bar{X})$ with $\pi(\alpha)=\pi(\beta)$, then $\alpha=\beta$. (This can also be verified directly, by a similar limiting argument.) It remains to show that such paths always exist:

Lemma 3.5.2. Any two points of $\bar{X}$ can be joined by a path in $\operatorname{geod}(\bar{X})$.

Proof. Suppose $x, y \in \bar{X}$. Since every constant path lies in $\operatorname{geod}(X)$, we can suppose that $x \neq y$. By Lemma 3.2.1, both $x$ and $y$ are accessible by smooth paths of finite length in $X$. From the geodesic convexity of $X$ (Proposition 3.4.2) and Lemma 3.3.1, we see that $x$ and $y$ can be joined by a path in $\operatorname{geod}(\bar{X})$. 
In summary, we have shown:

Proposition 3.5.3. For all $x, y \in \bar{X}$, there is a unique $\alpha \in \operatorname{geod}(\bar{X})$ with $\pi(\alpha)=(x, y)$. Moreover length $\alpha=d(x, y)$. In fact, $\alpha$ is the unique constant-speed globally length-minimising path in $\bar{X}$ from $x$ to $y$.

We can now use the term "geodesic" without ambiguity. As with $X$, we write $[x \rightarrow y]$ for the unique path in $\operatorname{geod}(\bar{X})$ joining $x$ to $y$. We write $[x, y] \subseteq \bar{X}$ for the image of $[x \rightarrow y]$. As before, $[x, y]=[y, x]$ and $[x, x]=\{x\}$. Note that for all $x, y \in \bar{X}$, we have $[x, y]=\{z \in \bar{X} \mid d(x, z)+d(z, y)=d(x, y)\}$.

Note that since $(\bar{X}, d)$ is $\operatorname{CAT}(0)$, it follows that the distance function is convex (cf. Lemma 3.4.6). In particular, geodesics vary continuously on their endpoints, and so:

Proposition 3.5.4. The map $\pi: \operatorname{geod}(\bar{X}) \longrightarrow \bar{X} \times \bar{X}$ is a homeomorphism.

We remark that if we fix one endpoint, then geodesics vary in a $C^{1}$ fashion:

Proposition 3.5.5. Given $a \in \bar{X}$, define $f_{a}: X \times(0,1) \longrightarrow X$ by $f_{a}(x, t)=$ $[a \rightarrow x](t)$. Then $f_{a}$ is $C^{1}$.

Proof. Clearly, if $a \in X$, then $f_{a}$ is smooth. If $a \in \bar{X} \backslash X$, we choose a sequence of points $a_{n} \in X$ with $a_{n} \rightarrow a$, and check that the derivatives of the functions $f_{a_{n}}$ converge. This can be done by considering Jacobi fields along $\left[x, a_{n}\right]$ (c.f. the case of horofunctions $[\mathbf{H e I}]$ ).

\section{The compactification.}

In this chapter, we assume that $X$ satisfies axioms (A) and (B). We shall describe the compactification $X_{C}=X \cup X_{I}$, where $X_{I}$ is the "ideal sphere". Thus, $X_{I}$ may be thought of, set theoretically, as the union of $X_{I}^{0} \equiv \bar{X} \backslash X$ and a set, $X_{I}^{\infty}$ of asymptote classes of geodesic rays. We shall show that $X_{C}$ is homeomorphic to a closed ball (Proposition 4.5.2.)

4.1. Geodesic rays. A geodesic ray based at $x \in \bar{X}$ is a path $\alpha:[0, \infty) \longrightarrow$ $\bar{X}$ such that $\alpha(0)=x$, and $\alpha((0, \infty)) \subseteq X$, and such that $\alpha \mid(0, \infty)$ is a geodesic parameterised by arc length.

We know (Proposition 3.5.3) that geodesics are length-minimising in $\bar{X}$. In particular, $\alpha$ must be a proper map.

Suppose $\alpha, \beta$ are geodesic rays. By Lemma 3.4.6, the map

$$
[t \mapsto d(\alpha(t), \beta(t))]
$$

is convex. Thus, if $d(\alpha(t), \beta(t))$ is bounded above, then

$$
d(\alpha(t), \beta(t)) \leq d(\alpha(0), \beta(0)) \quad \text { for all } t .
$$


Definition. We say that the rays $\alpha$ and $\beta$ are asymptotic if $d(\alpha(t), \beta(t))$ is bounded as $t \longrightarrow \infty$.

Clearly this is an equivalence relation on the set of geodesic rays. Note that:

Lemma 4.1.1. If $\alpha$ and $\beta$ are asymptotic rays, then the map

$$
[t \mapsto d(\alpha(t), \beta(t))]
$$

is monotonically non-increasing.

Corollary 4.1.2. Two asymptotic rays based at the same point are equal.

Proposition 4.1.3. Suppose that $\beta$ is a geodesic ray, and $x \in \bar{X}$. Then there is a (unique) geodesic ray based at $x$ asymptotic to $\beta$.

Proof. For this, we need only the convexity of the distance function (Lemma 3.4.6), and the completeness of $\bar{X}$.

For $n \in \mathbb{N}$, set $l_{n}=d(x, \beta(n))$. Let $\alpha_{n}:\left[0, l_{n}\right] \longrightarrow \bar{X}$ be the geodesic from $x$ to $\beta(n)$ parameterised by arc-length. Note that $n-l_{0} \leq l_{n} \leq n+l_{0}$. From Lemma 3.4.6 applied to $\beta$ and $\alpha_{n}$, we see that $d(\alpha(t), \beta(t)) \leq l_{0}$ provided $t \leq n-l_{0}$. Thus, if $m \geq n \geq l_{0}$, then $d\left(\alpha_{n}\left(n-l_{0}\right), \alpha_{m}\left(n-l_{0}\right)\right) \leq 2 l_{0}$. Now, by Lemma 3.4.6 applied to $\alpha_{n}$ and $\alpha_{m}$, we see that for all $t \in\left[0, n-l_{0}\right]$, we have $d\left(\alpha_{n}(t), \alpha_{m}(t)\right) \leq \frac{2 l_{0} t}{n-l_{0}}$. Thus, for a fixed $t$, the sequence $\left(\alpha_{n}(t)\right)$ is a Cauchy sequence, and so tends to a limit $\alpha(t) \in \bar{X}$. Now each $\alpha_{n}$ is length-minimising, and so $d(\alpha(t), \alpha(u))=|t-u|$ for all $t, u \in[0, \infty)$. Thus by Proposition 3.5.3, we see that $\alpha((0, \infty)) \subseteq X$ and $\alpha \mid(0, \infty)$ is geodesic. For all $n \geq t+l_{0}$, we have $d\left(\beta(t), \alpha_{n}(t)\right) \leq l_{0}$, and so $d(\alpha(t), \beta(t)) \leq l_{0}$. Thus $\alpha$ and $\beta$ are asymptotic.

Now, let $X_{I}^{\infty}$ be the set of asymptote classes of geodesic rays. We write $X_{I}^{0}$ for the set $\bar{X} \backslash X$, and define the ideal sphere, $X_{I}$, as a disjoint union $X_{I}=X_{I}^{0} \sqcup X_{I}^{\infty}$. We write $X_{C}=X \sqcup X_{I}$ for the compactification of $X$, and $\iota: \bar{X} \longrightarrow X_{C}$ for the natural inclusion. We shall describe the topology on these spaces in Section 4.3.

Suppose that $x \in \bar{X} \equiv X \cup X_{I}^{0}$ and that $y \in X_{I}^{\infty}$. Lemma 4.1 .3 tells us that there is a unique geodesic ray $\beta$ based at $x$ and in the class $y$. We say that $\beta$ tends to the point $y$. Write $[x, y]=\beta([0, \infty)) \cup\{y\} \subseteq X_{C}$, and refer to $[x, y]$ as the geodesic joining $x$ to $y$. Given the existence and uniqueness of geodesics in $\bar{X}$, we have established that:

Lemma 4.1.4. Given $(x, y) \in X_{C} \times X_{C} \backslash\left(X_{I}^{\infty} \times X_{I}^{\infty}\right)$, then there is a unique geodesic $[x, y]$ joining $x$ to $y$.

We may extend the notations $\overrightarrow{x y}$ and $y \hat{x} z$ to the case where $x \in X$ and $y, z \in X_{C} \backslash\{x\}$. 
Note that from the proof of Proposition 4.1.3, we see that if $z, x \in X$, $y \in X_{I}^{\infty}$, and $y_{n} \in[z, y] \cap X$ is a sequence of points tending to $y$, then the vectors $\overrightarrow{x y_{n}}$ tend to $\overrightarrow{x y}$ in the unit tangent space at $x$.

If we fix $y \in X_{I}^{\infty}$, then the vector field $[x \mapsto \vec{x} \vec{y}]: X \longrightarrow T X$, is $C^{1}$, where $T X$ is the total tangent bundle to $X$. This may be proven using the convergence of Jacobi fields just as in the complete case. We may also define a positive-time flow $\phi: X \times[0, \infty) \longrightarrow X$ along this field. Thus, $\phi(x, t)=\beta(t)$, where $\beta$ is the geodesic ray based at $x$ tending to $y$. As in the complete case, we have:

Proposition 4.1.5. The flow $\phi: X \times[0, \infty) \rightarrow X$ is $C^{2}$.

4.2. Horofunctions. In this section, we describe the "horofunctions" (or "Busemann functions") about a point $y \in X_{I}^{\infty}$. The results will be used again in Chapter 6, though, for the moment, it is something of a digression.

Fix $y \in X_{I}^{\infty}$. Suppose $a \in \bar{X}$. Let $\beta$ be the geodesic ray based at $a$ tending to $y$. Given any $x \in \bar{X}$, the function $[t \mapsto t-d(x, \beta(t))]$ is monotonically increasing in $t$. Moreover it is bounded above (by $d(x, a)$ ). It thus tends to a well-defined limit $h_{a}(x)=\lim _{t \rightarrow \infty}(t-d(x, \beta(t)))$. We see easily that $\left|h_{a}(x)-h_{a}\left(x^{\prime}\right)\right| \leq d\left(x, x^{\prime}\right)$ for all $x, x^{\prime} \in \bar{X}$. Thus, $h_{a}: \bar{X} \longrightarrow \mathbb{R}$ is continuous. Also, one can show that $h_{a}$ is $C^{2}$. This follows as in the complete case (see $[\mathbf{H e I}])$. We refer to $h_{a}$ as a horofunction about $y$.

To see that $h_{a}$ is at least $C^{1}$ on $X$ is elementary. For a fixed $t$, write $f_{t}(x)=$ $t-d(x, \beta(t))$. Thus $f: X \longrightarrow \mathbb{R}$ is smooth on $X$, and its gradient, $\operatorname{grad} f_{t}$ at $x$ equals $\overrightarrow{x y}_{t}$ where $y_{t}=\beta(t)$. From the Angle Comparison Theorem (Proposition 3.4 .4 ) we can verify that $\overrightarrow{x y_{t}}$ tends to $\overrightarrow{x y}$ as $t \rightarrow \infty$. Moreover, this convergence is uniform on compact subsets of $X$. Thus $f$ is $C^{1}$, and $\operatorname{grad} f(x)=\overrightarrow{x y}$.

As a consequence, we may deduce that any two horofunctions about $y$ differ by a constant.

Lemma 4.2.1. If $a, b, x \in \bar{X}$, then $h_{b}(x)=h_{b}(a)+h_{a}(x)$.

Proof. From the previous paragraph, we know that for all $x \in X$, we have $\operatorname{grad}\left(h_{b}-h_{a}\right)(x)=0$, and so $h_{b}-h_{a}$ is constant on $X$. By continuity, it is constant on all of $\bar{X}$. Since $h_{a}(a)=0$, we must have $h_{b}(x)-h_{a}(x)=h_{b}(a)$ as required.

We remark that we do not really need the differentiable structure on $X$ in order to deduce Lemma 4.2.1. In fact, it follows from the $\operatorname{CAT}(0)$ inequality. The important observation is that if we have a "long" rectangle in a CAT(0)space, then the sum of the two diagonals is approximately equal to the sum 
of the two long edges. More specifically, suppose $x, y, z, w \in \bar{X}$, are any four points, then $|d(x, y)+d(z, w)-d(y, z)-d(x, w)| \leq \frac{1}{R}\left(d(x, z)^{2}+d(y, w)^{2}\right)$, where $R=\min (d(x, y), d(z, w), d(y, z), d(x, w))$. Here $x z$ and $y w$ are the "short" sides. The exact form of the right-hand term of the inequality is unimportant. We just need to note that if the rectangle is sufficiently long, while the lengths of the short sides remain bounded, then the first term can be made arbitrarily small. We leave the reader to work out the details of this, and relate it to the definition of horofunctions.

Suppose that $h$ is a horofunction about $y$. We have seen that $|\operatorname{grad} h|=1$ everywhere, and so the level sets of $h$ give us a codimension- 1 foliation of $X$ by $C^{2}$ submanifolds. Given $t \in \mathbb{R}$, write $S(t)=X \cap h^{-1}(t)$. We refer to $S(t)$ as a horosphere about $y$. Let $B(t)=X \backslash h^{-1}([t, \infty))$. Thus $B(t)$ is a closed convex subset of $X$ with boundary $S(t)$. We call $B(t)$ a horoball about $t$.

Given a horoball $B$ about $y$, we may define the nearest point retraction $\rho$ of $X$ onto $B$. Thus, for all $x \in X, \rho(x)$ is the nearest point on $[x, y] \cap B$ to $x$. We see that $\rho(x)=x$ for all $x \in B$, and $\rho(X \backslash B)=S=\partial B$. We have observed that $S$ is a $C^{2}$-submanifold. We have

Lemma 4.2.2. The nearest point retraction $\rho \mid(X \backslash B): X \backslash B \longrightarrow S$ is $C^{2}$.

Proof. Let $h$ be the horofunction with $h(S)=\{0\}$. Apply Proposition 4.1.5, noting that $\rho(x)=\phi(x,-h(x))$ for all $x \in X \backslash B$.

4.3. The compactified topology. Choose any basepoint $p \in X$, and let $T_{p}^{1}(X)$ be the unit tangent space at $p$. Now each vector in $T_{p}^{1}(X)$ determines the germ of a geodesic emanating from $p$. We may continue this geodesic until either we arrive at some point of $X_{I}^{0}$, or until we form a geodesic ray tending to some point of $X_{I}^{\infty}$. Lemma 4.1 .4 thus gives an identification of $X_{I}=X_{I}^{0} \cup X_{I}^{\infty}$ with $T_{p}^{1}(X)$. Thus, $X_{I}$ is given the topology of an $(n-1)$ sphere. This topology turns out to be independent of the choice of basepoint $p \in X$. Moreover, it may be extended to give $X_{C}$ the topology of a closed $n$-ball. In this, and the next two sections we give an account of this.

The identification $\bar{X} \equiv X \cup X_{I}^{0} \subseteq X_{C}$ gives us a metric $d$ on $X \cup X_{I}^{0}$. We may extend this to a map $d: X_{C} \times X_{C} \longrightarrow[0, \infty]$ by setting $d(x, x)=0$ and $d(x, y)=\infty$ when $x \in X_{I}^{\infty}$ and $y \in X_{C} \backslash\{x\}$. Given $x \in X \cup X_{I}^{0}$, and $r \geq 0$, we write

$$
N(x, r)=\left\{y \in X_{C} \mid d(x, y) \leq r\right\}
$$

If $p \in X$, write

$$
C(p, x, r)=\left\{y \in X_{C} \mid d(x,[p, y]) \leq r\right\}
$$


In other words, $y \in C(p, x, r)$, if and only if $[p, y]$ meets $N(x, r)$. Clearly $N(x, r) \subseteq C(p, x, r)$. The following is a simple consequence of the CAT(0) inequality.

Lemma 4.3.1. Suppose that $p \in X$ and $y \in X_{I}^{\infty}$. Given $z \in[p, y] \cap X$, and $r, r^{\prime}>0$, then there is some $w \in[p, y] \cap X$ such that $C\left(p, w, r^{\prime}\right) \subseteq C(p, z, r)$.

We may now define a topology, $\tau\left(X_{C}, p\right)$, on $X_{C}$, relative to the point $p \in X$. We describe neighbourhood bases for points $y \in X_{C}$ as follows. If $y \in X$, we take as neighbourhood base the collection $\{N(y, \epsilon) \mid \epsilon>0\}$. If $y \in X_{I}^{0}$, we take as neighbourhood base $\{C(p, y, \epsilon) \mid \epsilon>0\}$. If $y \in X_{I}^{\infty}$, we take as neighbourhood base $\{C(p, x, \epsilon) \mid x \in[p, y] \cap X, \epsilon>0\}$. Note that, in the last case, by Lernma 4.3.1, we could equally well take as neighbourhood base $\{C(p, x, r) \mid x \in[p, y] \cap X\}$ for any fixed $r>0$. It is easily verified that these sets form the basis for a topology $\tau\left(X_{C}, p\right)$ on $X_{C}$. Clearly, its restriction to $X$ agrees with the metric topology. However, its restriction to $X \cup X_{I}^{0} \equiv \bar{X}$ is, in general, coarser than the metric topology. We aim to show that $\tau\left(X_{C}, p\right)$ is independent of $p \in X$. The following lemma will be used in several places in the rest of this paper.

Lemma 4.3.2. Given $a \in X_{I}^{0}$, and $h, \eta>0$, we can find $r>0$ with the following property. Suppose $(y, z) \in\left(X_{C} \times X_{C}\right) \backslash\left(X_{I}^{\infty} \times X_{I}^{\infty}\right)$ and $x \in$ $N(a, r) \cap X$. If $d(a,[y, z]) \geq h$, then $y \hat{x} z \leq \eta$.

Proof. By hypothesis (B), we can find $K, h_{0}>0$ such that if $d(x, a) \leq h_{0}$, then $\kappa(x) \leq-1 / K^{2} d(x, a)$. Suppose $h, \eta>0$. Let $r>0$, depending on $h$ and $\eta$, be as determined below. We can assume that $r<h^{\prime}=\min \left(h, h_{0}\right)$. Let $R=h^{\prime}-r$.

Now let $x, y, z$ be as in the statement of the lemma. For the moment, we assume that $y, z \in X \cup X_{I}^{0}$. The general case will follow by continuity. We want that $y \hat{x} z \leq \eta$.

Since $d(a,[y, z]) \geq h$, we have that $x \notin[y, z]$. Let $\theta=y \hat{x} z$. We can suppose that $\theta>0$. Now, $x, y, z$ are the vertices of a ruled surface obtained by joining $x$ to each point $w \in[y, z]$ by a geodesic $[x, w]$. Thus $P=\bigcup\{[x, w] \mid w \in[y, z]\}$ is a non-positively curved 3-gon. In fact, if $q$ lies in int $P=P \backslash \partial P$, then the intrinsic curvature $\kappa_{P}(q)$ is at most $\kappa(q)$. By Gauss-Bonnet, we find that

$$
-\int_{P} \kappa(q) d \omega(q) \leq-\int_{P} \kappa_{P}(q) d \omega(q) \leq \pi
$$

where $d \omega$ is the area element of $P$.

Suppose $t \in(0, R)$, and $w \in[y, z] \backslash\{y, z\}$. Let $q(w, t)$ be the point of $[x, w]$ with $d(x, q(w, t))=t$. (Figure 4a.) Now $d(a, q(w, t)) \leq d(x, a)+$ 
$d(x, q(w, t)) \leq r+t$. Thus $-\kappa(q(w, t)) \geq 1 / K^{2} d(a, q(w, t))^{2} \geq 1 / K^{2}(r+t)^{2}$. By the Angle Comparison Theorem (Proposition 3.4.4), we see that the path traced out by $q(w, t)$ as $w$ moves on $[y, z]$ has length at least $\theta t$. Thus,

$$
\begin{aligned}
\pi & \geq-\int_{P} \kappa(p) d \omega(q) \\
& \geq \int_{0}^{R} \frac{\theta t}{K^{2}(r+t)^{2}} d t \\
& =\frac{\theta}{K^{2}}\left(-\log \left(r / h^{\prime}\right)+\left(r / h^{\prime}\right)-1\right) \\
& =\frac{\theta}{K^{2}} f\left(r / h^{\prime}\right)
\end{aligned}
$$

where $f(s)=s-\log s-1$. Now $f(s) \rightarrow \infty$ as $s \rightarrow 0$, and so if $r / h^{\prime}$ is sufficiently small, we have $\theta \leq \pi K^{2} / f\left(r / h^{\prime}\right) \leq \eta$ as required.

To deal with the case where $y \in X_{I}^{\infty}$ and $z \in X \cup X_{I}^{0}$, choose a sequence of points $y_{n} \in[y, z] \cap X$ with $y_{n} \rightarrow y$. As observed in Section 4.1, we have $\overrightarrow{x y_{n}} \rightarrow \overrightarrow{x y}$, and so the general case follows by continuity.

Proposition 4.3.3. The topology $\tau\left(X_{C}, p\right)$ is independent of $p \in X$.

Proof. Suppose $p, p^{\prime} \in X$. Certainly $\tau\left(X_{C}, p\right)$ and $\tau\left(X_{C}, p^{\prime}\right)$ agree on $X$. We thus want to show that for all $y \in X_{I}$, the neighbourhood bases with respect to $p$ and $p^{\prime}$, as described above, are equivalent.

Suppose, first, that $y \in X_{I}^{\infty}$. Let $l=d\left(p, p^{\prime}\right)$ and suppose $r>0$. Given $x \in[p, y] \cap X$, we want to find $x^{\prime} \in\left[p^{\prime}, y\right] \cap X$ with $C\left(p^{\prime}, x^{\prime}, r\right) \subseteq C(p, x, r)$. By Lemma 4.3.1, we have $z \in[p, y]$ so that $C(p, z, r+2 l) \subseteq C(p, x, r)$. By Lemma 4.1.1, we can find $x^{\prime} \in\left[p^{\prime}, y\right]$ with $d\left(z, x^{\prime}\right) \leq l$. If $w \in C\left(p^{\prime}, x^{\prime}, r\right)$ so that $d\left(x^{\prime},\left[p^{\prime}, w\right]\right) \leq r$, then the $\operatorname{CAT}(0)$ inequality, applied to the triangle $w p p^{\prime}$, tells us that $d\left(x^{\prime},[p, w]\right) \leq d\left(x^{\prime},\left[p^{\prime}, w\right]\right)+d\left(p, p^{\prime}\right) \leq r+l$. Thus $d(z,[p, w]) \leq(r+l)+d\left(z, x^{\prime}\right) \leq r+2 l$, and so $w \in C(p, z, r+2 l)$. We have shown that $C\left(p^{\prime}, x^{\prime}, r^{\prime}\right) \subseteq C(p, x, r)$ as required.

Now suppose that $y \in X_{I}^{0}$. Given $\epsilon>0$, we want to find $\epsilon^{\prime}>0$ so that $C\left(p^{\prime}, y, \epsilon^{\prime}\right) \subseteq C(p, y, \epsilon)$. We can assume that $\epsilon<d\left(y, p^{\prime}\right)$. Let $h_{0}=$ $d\left(y,\left[p, p^{\prime}\right]\right)$ and $h=\min \left(h_{0}, \epsilon\right)$. Lemma 4.3.2 gives us some $\epsilon^{\prime}>0$ such that if $x \in N\left(y, \epsilon^{\prime}\right) \cap X$ and $(a, b) \in\left(X_{C} \times X_{C}\right) \backslash\left(X_{I}^{\infty} \times X_{I}^{\infty}\right)$, then $d(y,[a, b]) \leq h$ or $a \hat{x} b \leq \pi / 3$. Now suppose that $z \in C\left(p^{\prime}, y, \epsilon^{\prime}\right)$, so that there some $x \in$ $\left[p^{\prime}, z\right] \cap N\left(y, \epsilon^{\prime}\right) \cap X$. Since $d\left(y,\left[p, p^{\prime}\right]\right) \geq h$, we have $p \hat{x} p^{\prime} \leq \pi / 3$. Thus $p \hat{x} z \geq \pi-\pi / 3=2 \pi / 3$ and so $d(y,[p, z]) \leq h \leq \epsilon$. Thus $z \in C(p, y, \epsilon)$. We have shown that $C\left(p^{\prime}, y, \epsilon^{\prime}\right) \subseteq C(p, y, \epsilon)$.

We shall write $\tau\left(X_{C}\right)$ for the topology thus defined on $X_{C}$. The following is easily verified. 
Proposition 4.3.4. The natural inclusion $\iota: \bar{X} \longrightarrow X_{C}$ is continuous.

Here, and in the rest of this paper, we adopt the convention that $\bar{X}$ has the metric topology, whereas $X \cup X_{I}^{0}$ has the subspace topology induced from $\tau\left(X_{C}\right)$.

It is not very hard to see that $\left(X_{C}, \tau\left(X_{C}\right)\right)$ is compact hausdorff. We shall not give a direct proof here, since we show, in the next two sections, that it is homeomorphic to a closed $n$-dimensional ball.

4.4. Starlike sets. Let $\mathbb{E}^{n}$ be $n$-dimensional euclidean space, and let $\underline{0} \in \mathbb{E}^{n}$ be any point. We identify the unit tangent space $T_{0}^{1} \mathbb{E}^{n}$ with the unit sphere $S^{n-1}$. We may identify $\mathbb{E}^{n}$ with $\left(S^{n-1} \times[0, \infty)\right) / \sim$, where $(\xi, 0) \sim(\zeta, 0)$ for all $\xi, \zeta \in S^{n-1}$, otherwise equivalence classes are single points. We may identify the compactified space $\mathbb{E}_{C}^{n}$ with $\left(S^{n-1} \times[0, \infty]\right) / \sim$. We write $\langle\xi, t\rangle$ for the $\sim$-class of $(\xi, t)$. Thus, $\underline{0}=\langle\xi, 0\rangle$ for all $\xi \in S^{n-1}$.

Note that a subset $\Sigma \subseteq \mathbb{E}^{n}$ is open and starlike about $\underline{0}$ if and only if it has the form $\{\langle\xi, t\rangle \mid 0 \leq t<f(\xi)\}$, where $f: S^{n-1} \longrightarrow(0, \infty]$ is lowersemicontinuous. We write

$$
\Sigma_{C}=\left\{\langle\xi, t\rangle \in \mathbb{E}_{C}^{n} \mid 0 \leq t \leq f(\xi)\right\} .
$$

Thus, $\Sigma_{C}$ is also starlike about $\underline{0}$, and a subset of the closure of $\Sigma$ in $\mathbb{E}_{C}^{n}$. Write $\Sigma_{I}=\Sigma_{C} \backslash \Sigma=\left\{\langle\xi, t\rangle \in \mathbb{E}_{C}^{n} \mid t=f(\xi)\right\}$. (Note that this notation is consistent with that previously defined if $\Sigma=\mathbb{E}^{n}=X$.) We put a topology $\tau\left(\Sigma_{C}\right)$ on $\Sigma_{C}$ as follows. We demand that the subspace topology on $\Sigma$ induced from $\tau\left(\Sigma_{C}\right)$ agrees with that induced from $\mathbb{E}^{n}$. If $\langle\xi, t\rangle \in \Sigma_{I}$, we take as a base of neighbourhoods the collection $\{D(U, u)\}$ where

$$
D(U, u)=\left\{\langle\zeta, v\rangle \in \Sigma_{C} \mid \zeta \in U, v \geq u\right\}
$$

and $U$ ranges over all neighbourhoods of $\zeta$ in $\zeta$, and $u$ ranges over the interval $(0, t)$. Thus, in general, the topology $\tau\left(\Sigma_{C}\right)$ on $\Sigma_{C}$ is coarser than the subspace topology induced from $\left(\mathbb{E}_{C}^{n}, \tau\left(\mathbb{E}_{C}^{n}\right)\right.$ ). (Note that $\tau\left(\mathbb{E}_{C}^{n}\right)$ agrees with our previous definition with $\Sigma=\mathbb{E}^{n}=X$.)

Now, if $a, b \in(0, \infty]$ and $h:[0, a] \longrightarrow[0, b]$ is a homeomorphism, with $h(0)=0$, then the map $\hat{h}=[\langle\xi, t\rangle \mapsto\langle\xi, h(t)\rangle]$ gives a homeomorphism of the ball $N(\underline{0}, a)$ onto $N(\underline{0}, b)$ (where $N(\underline{0}, \infty)=\mathbb{E}_{C}^{n}$ ). Moreover, if $\Sigma \subseteq N(\underline{0}, a) \subseteq$ $\mathbb{E}^{n}$ is open and starlike about $\underline{0}$, then so is $\Sigma^{\prime}=\hat{h}(\Sigma)$. Also, $\Sigma_{C}^{\prime}=\underline{\hat{h}}\left(\Sigma_{C}\right)$, and $\hat{h} \mid \Sigma_{C}:\left(\Sigma_{C}, \tau\left(\Sigma_{C}\right)\right) \longrightarrow\left(\Sigma_{C}^{\prime}, \tau\left(\Sigma_{C}^{\prime}\right)\right)$ is a homeomorphism.

Write $B^{n}$ for the closed unit $n$-ball (as a manifold), and write int $B^{n}=$ $B^{n} \backslash \partial B^{n}$ for its interior.

Lemma 4.4.1. Suppose $\Sigma \subseteq \mathbb{E}^{n}$ is open and starlike. Then, the pair 
$\left(\Sigma_{C}, \Sigma\right)$, with the topology given by $\tau\left(\Sigma_{C}\right)$, is homeomorphic to the pair $\left(B^{n}\right.$, int $\left.B^{n}\right)$.

Proof. Let $\mathbb{E}_{\infty}^{n}=\mathbb{E}^{n} \cup\{\underline{\infty}\}$ be the one point compactification of $\mathbb{E}^{n}$. Let $B_{0}=N(\underline{0}, 1) \subseteq \mathbb{E}^{n} \subseteq \mathbb{E}_{\infty}^{n}$, be the unit ball about $\underline{0}$. From the discussion prior to the statement of the lemma, we see that we can assume that $\Sigma \subseteq B_{0}$.

Let $g: \mathbb{E}^{n} \longrightarrow \mathbb{E}_{\infty}^{n} \backslash\{\underline{0}\}$ be the inversion given by $g(\langle\xi, t\rangle)=\langle\xi, 1 / t\rangle$ for $t>0$ and $g(\underline{0})=\underline{\infty}$. Restricted to $B_{0}$, the map $g$ gives a homeomorphism of $B_{0}$ onto $B_{\infty}=\mathbb{E}_{\infty}^{n} \backslash \operatorname{int} B_{0}$. Let $\Omega=g(\Sigma)$, and $\Omega_{C}=g\left(\Sigma_{C}\right)$. Let $\partial \Omega$ be the topological boundary of $\Omega$ in $\mathbb{E}_{\infty}^{n}$, so that $\partial \Omega \subseteq B_{\infty} \backslash\{\underline{\infty}\}$ and $\Omega_{C}=\Omega \cup \partial \Omega$. We define the map $\rho: \Omega_{C} \backslash\{\underline{\infty}\} \longrightarrow[0, \infty)$ by $\rho(x)=d_{\text {euc }}(x, \partial \Omega)$, where $d_{\text {euc }}$ is the euclidean distance.

Certainly, $\rho$ is continuous on $\Omega$, and $\rho(x)=0$ if and only if $x \in \Omega_{C} \backslash \Omega$. Moreover, if $\langle\xi, t\rangle,\langle\xi, u\rangle \in \Omega$ with $t<u$, then $\rho(\langle\xi, t\rangle)<\rho(\langle\xi, u\rangle)$. We now define $h: \Omega_{C} \longrightarrow \mathbb{E}_{C}^{n}$ by $h(\langle\xi, t\rangle)=\langle\xi, 1+\rho(\langle\xi, t\rangle)\rangle$ and $h(\underline{\infty})=\underline{\infty}$. Clearly, $h$ maps $\Omega_{C}$ bijectively onto $B_{\infty}$, and $h \mid \Omega$ is a homeomorphism onto int $B_{\infty}$. It follows that $j=g^{-1} h g$ maps $\Sigma_{C}$ bijectively onto $B_{0}$, and that $j \mid \Sigma$ is a homeomorphism onto int $B_{0}$. Moreover, a simple exercise shows that $j$ is, in fact, a homeomorphism from $\left(\Sigma_{C}, \tau\left(\Sigma_{C}\right)\right)$ to $B_{0}$.

With a bit more work, one can make a stronger statement, namely:

Lemma 4.4.2. Suppose that $\Sigma \subseteq \mathbb{E}^{n}$ is open and starlike. Then, there is a homeomorphism of $\left(\Sigma_{C}, \Sigma\right)$ to $\left(B^{n}\right.$, int $\left.B^{n}\right)$ whose restriction to $\Sigma$ is a smooth diffeomorphism onto int $B^{n}$.

Proof (Sketch). One way to do this is to approximate the map $\rho$, from the proof of Lemma 4.4.1, by a smooth map, $\rho^{\prime}$, with $\partial \rho^{\prime} / \partial t>0$ everywhere on $\Omega \backslash\{\underline{\infty}\}$. Define $\sigma: B_{\infty} \backslash\{\underline{\infty}\} \longrightarrow(0, \infty)$ by $\rho(\langle\xi, \sigma(\langle\xi, t\rangle)\rangle)=t$. We want to smooth out $\sigma$ on int $B_{0} \backslash\{\underline{\infty}\}$ to get a smooth map $\sigma^{\prime}$ with $\partial \sigma^{\prime} / \partial t>$ 0 . Given any positive integer $n$, define $\sigma_{n}: S^{n-1} \longrightarrow(0, \infty)$ by $\sigma_{n}(\xi)=$ $\sigma(\langle\xi, 1+1 / n\rangle)$. We approximate each $\sigma_{n}$ by a smooth map $\sigma_{n}^{\prime}: S^{n-1} \longrightarrow$ $(0, \infty)$ so that $\left|\sigma_{n}^{\prime}(\xi)-\sigma_{n}(\xi)\right| \leq 1 / 2 n(n+1)$ for all $\xi \in S^{n-1}$. In this way, we arrange that $\sigma_{n+1}^{\prime}(\xi)<\sigma_{n}(\xi)$ for all $\xi \in S^{n-1}$. By interpolation, we get a smooth function $\sigma^{\prime}: B(\underline{0}, 2) \backslash B_{0} \longrightarrow(0, \infty)$ so that $\sigma^{\prime}(\langle\xi, 1+1 / n\rangle)=\sigma_{n}^{\prime}(\xi)$ and $\partial \sigma^{\prime} / \partial t>0$. We now extend to a smooth function $\sigma^{\prime}:$ int $B_{\infty} \backslash\{\underline{\infty}\} \longrightarrow$ $(0, \infty)$ so that $\partial \sigma^{\prime} / \partial t>0$ everywhere, and $\sigma^{\prime}(\langle\xi, t\rangle)=t$ for all sufficiently large $t$. The identity $\rho^{\prime}\left(\left\langle\xi, \sigma^{\prime}(\langle\xi, t\rangle)\right\rangle\right)=t$ allows us to define a smooth $\operatorname{map} \rho^{\prime}: \Omega \backslash\{\underline{\infty}\} \longrightarrow(0, \infty)$, with $\partial \rho^{\prime} / \partial t>0$. We extend $\rho^{\prime}$ to a map $\Omega_{C} \backslash\{\underline{\infty}\} \rightarrow[0, \infty)$ by setting $\rho^{\prime}\left(\Omega_{C} \backslash \Omega\right)=\{0\}$. We now proceed as in Lemma 4.4.1. It may be verified that the map $j^{\prime}: \Sigma_{C} \longrightarrow B_{0}$ thus defined is a diffeomorphism on $\Sigma$. 
4.5. The logarithm map. In this section, we relate the discussion of starlike sets to our compactified manifold $X_{C}$.

Choose any point $p \in X$ and then identify the unit tangent space $T_{p}^{1}(X)$ with $S^{n-1}$ via an isometry $\phi: T_{p}^{1}(X) \longrightarrow S^{n-1}$. Recall the description of $\mathbb{E}_{C}^{n}$ as a quotient of $S^{n-1} \times[0, \infty]$, given in the previous section. We define a map $\log : X_{C} \longrightarrow \mathbb{E}_{C}^{n}$ as follows. Set $\log (p)=\underline{0}$, and for $x \in X_{C} \backslash\{p\}$, set $\log (x)=\langle\phi(\overrightarrow{p x}), d(p, x)\rangle$, were $d(p, x)=\infty$ for $x \in X_{I}^{\infty}$. By Lemma 4.1.4, we see that $\log$ is a bijection onto its image $\Sigma_{C}(X)=\log \left(X_{C}\right) \subseteq \mathbb{E}_{C}^{n}$. Moreover $\log \mid X$ gives a diffeomorphism of $X$ onto $\Sigma(X)=\log (X) \subseteq X$. This follows as in the complete case. Thus, $\Sigma$ is open and starlike about $\underline{0}$. Also, we have that, set theoretically, $(\Sigma(X))_{C}=\Sigma_{C}(X)$.

Lemma 4.5.1. The map $\log :\left(X_{C}, \tau\left(X_{C}\right)\right) \longrightarrow\left(\Sigma_{C}(X), \tau\left(\Sigma_{C}(X)\right)\right)$ is a homeomorphism.

Proof. The fact that $\log$ is continuous is a simple consequence of the Angle Comparison Theorem (Proposition 3.4.4). We have also noted that $\log \mid X$ is a diffeomorphism. It remains therefore to show that $\exp =\log ^{-1}$ : $\Sigma_{C}(X) \longrightarrow X_{C}$ is continuous at all points of $\Sigma_{C}(X) \backslash \Sigma(X)$.

Suppose that $y=\exp (\langle\xi, t\rangle) \in X_{I}^{0}$. Given $r \in(0, t)$, let $x=\exp (\langle\xi, t-r / 2\rangle)$. Thus, $x \in[p, y]$ with $d(x, y)=r / 2$. By the continuity of $\exp \mid X$, we can find $U \subseteq S^{n-1}$ which is a neighbourhood of $\xi$, such that if $\xi^{\prime} \in U$, then $\langle\xi, t-r / 2\rangle \in \Sigma$ and $d(x, \exp (\langle\xi, t-r / 2\rangle)) \leq r / 2$. It follows that $d\left(y, \exp \left(\left\langle\xi^{\prime}, t-r / 2\right\rangle\right)\right) \leq r / 2$, and so $\exp \left(\left\langle\xi^{\prime}, t^{\prime}\right\rangle\right) \in C(p, y, r)$ whenever $t^{\prime} \geq t-r / 2$ and $\left\langle\xi^{\prime}, t^{\prime}\right\rangle \in \Sigma_{C}(X)$. This shows that $\exp (D(U, t-r / 2)) \subseteq$ $C(p, y, r)$, and so exp is continuous at $\langle\xi, t\rangle$.

The case where $\exp (\langle\xi, t\rangle) \in X_{I}^{\infty}$ is similar.

Putting Lemma 4.5.1 together with Lemma 4.4.2, we have:

Proposition 4.5.2. The pair $\left(X_{C}, X\right)$, in the topology $\tau\left(X_{C}\right)$, is homeomorphic to the pair $\left(B^{n}\right.$, int $\left.B^{n}\right)$ where $B^{n}$ is the unit $n$-dimensional ball, and int $B^{n}$ is its interior. Moreover, we can arrange that the homeomorphism restricted to $X$ gives a smooth diffeomorphism onto int $B^{n}$.

In particular, we see that $X_{C}$ is compact metrisable.

\section{Continuity properties.}

As in the previous chapter, we are assuming that $X$ satisfies axioms (A) and (B). Our aim here is to investigate how geodesics move as we vary the endpoints. 
5.1. Lower semicontinuity of the distance function. We extend the metric $d$ on $\bar{X} \equiv X \cup X_{I}^{0}$ to a map $d: X_{C} \times X_{C} \longrightarrow[0, \infty]$ by setting $d(x, x)=0$ and $d(x, y)=\infty$ if $x \in X_{I}^{\infty}$ and $y \in X_{C} \backslash\{x\}$. We claim that this map is lower-semicontinuous on $X_{C} \times X_{C}$ given the product topology $\tau\left(X_{C}\right) \times \tau\left(X_{C}\right)$.

Lemma 5.1.1. Suppose that $x, y \in X \cup X_{I}^{0}$ and $x \neq y$. Given and $h>0$, there exist neighbourhoods $U$ of $x$ and $V$ of $y$ in $\tau\left(X_{C}\right)$ such that if $u \in U$ and $v \in V$, and $(u, v) \notin X_{I}^{\infty} \times X_{I}^{\infty}$, then $d(x,[u, v]) \leq h$ and $d(y,[u, v]) \leq h$. In fact, we can find $u^{\prime}, v^{\prime} \in[u, v]$ with $d\left(x, u^{\prime}\right) \leq h, d\left(y, v^{\prime}\right) \leq h$ and $u^{\prime} \in\left[u, v^{\prime}\right]$.

Proof. We shall deal with the case where $x$ and $y$ both lie in $X_{I}^{0}$. The remaining cases are simpler. We can assume that $h<\frac{1}{4} d(x, y)$. By Lemma 4.3.2, there is some $\epsilon_{1}>0$ such that if $a \in N\left(x, \epsilon_{1}\right) \cap X$ and $(z, w) \in$ $\left(X_{C} \times X_{C}\right) \backslash\left(X_{I}^{\infty} \times X_{I}^{\infty}\right)$, then either $d(x,[z, w]) \leq h$, or else $z \hat{a} w \leq \pi / 2$. There is a similar constant $\epsilon_{2}$ corresponding to $b$. Let $\epsilon=\min \left(\epsilon_{1}, \epsilon_{2}, h\right)$. Let $U=C(y, x, \epsilon)$ and $V=C(x, y, \epsilon)$. From the definition $\tau\left(X_{C}\right)=\tau\left(X_{C}, x\right)=$ $\tau\left(X_{C}, y\right)$ we see that $U, V$ are neighbourhoods of $x, y$ respectively in $\tau\left(X_{C}\right)$. Suppose that $u \in U$ and $v \in V$, so that $d(x,[u, y]) \leq \epsilon$ and $d(y,[v, x]) \leq$ $\epsilon$. Choose $a \in[u, y] \cap N(x, \epsilon) \cap X$ and $b \in[v, x] \cap N(x, \epsilon) \cap X$. By the Angle Comparison Theorem (Proposition 3.4.4), we see that $a \hat{b} x \leq \pi / 3$, and so $a \hat{b} v \geq 2 \pi / 3>\pi / 2$. Thus $d(y,[a, v]) \leq h$. So, again by the Angle Comparison Theorem, we have vây $\leq \pi / 3$ and so $u \hat{a} v \geq 2 \pi / 3>\pi / 2$. Thus $d(x,[u, v]) \leq h$. Similarly, $d(y,[u, v]) \leq h$.

Note that $d(x, y) \geq d(u, x)+d(x, y)-2 \epsilon$. Thus if $u^{\prime}, v^{\prime} \in[u, v]$ with $d\left(x, u^{\prime}\right) \leq h$ and $d\left(y, v^{\prime}\right) \leq h$ then $d\left(u, u^{\prime}\right) \geq d\left(u, v^{\prime}\right)+d(x, y)-2 \epsilon-2 h \geq$ $d\left(u, v^{\prime}\right)$ since $\epsilon \leq h$ and $4 h \leq d(x, y)$. If $u \notin X_{I}^{\infty}$, this shows that $u^{\prime} \in\left[u, v^{\prime}\right]$. If $u \in X_{I}^{\infty}$, choose $u_{0} \in\left[u, u^{\prime}\right] \cap\left[u, v^{\prime}\right] \cap X$, and apply the same argument with $u_{0}$ replacing $u$.

Lemma 5.1.2. Suppose $x \in X_{I}^{0}, y \in X_{I}^{\infty}$ and $z \in[x, y] \cap X$. Given $h>0$, then there is some $\epsilon>0$ and a neighbourhood $V$ about $y$ in $\tau\left(X_{C}\right)$ such that if $u \in N(x, \epsilon)$ and $v \in V$, then $d(z,[u, v]) \leq h$.

Proof. Take $\epsilon=h / 2$. By the definition of $\tau\left(X_{C}\right)$, the set $V=C(x, z, \epsilon)$ is a neighbourhood of $y$. Suppose $v \in V$ and $u \in N(x, \epsilon)$. Then $d(z,[x, v]) \leq \epsilon$, and so by $\operatorname{CAT}(0)$ applied to $x u v$, we find that $d(z,[u, v]) \leq 2 \epsilon \leq h$.

Lemma 5.1.3. Suppose $x \in X \cup X_{I}^{0}$ and $y \in X_{I}^{\infty}$ and $z \in[x, y] \cap X$. Given any $h>0$, there are neighbourhoods $U$ of $x$ and $V$ of $y$ in $\tau\left(X_{C}\right)$ such that if $u \in U$ and $v \in V$ and $(u, v) \notin X_{I}^{\infty} \times X_{I}^{\infty}$, then $d(x,[u, v]) \leq h$ and $d(z,[u, v]) \leq h$. Moreover, we can find $u^{\prime}, v^{\prime} \in[u, v]$ with $d\left(x, u^{\prime}\right) \leq h$ and 
$d\left(z, v^{\prime}\right) \leq h$ and $u^{\prime} \in\left[u, v^{\prime}\right]$.

Proof. As with Lemma 5.1.1. Use Lemma 5.1.2.

Proposition 5.1.4. The map $d: X_{C} \times X_{C} \longrightarrow[0, \infty]$ is lower semicontinuous, where $X_{C} \times X_{C}$ is given the product topology $\tau\left(X_{C}\right) \times \tau\left(X_{C}\right)$.

Proof. Suppose $x, y \in X_{C}$. If $x=y$, then $d(x, y)=0$ and there is nothing to prove. If $x \in X \cup X_{I}^{0}$ and $y \in X_{C} \backslash\{x\}$, the result follows from Lemmas 5.1.1 and 5.1.3. The only remaining case is where $x, y \in X_{I}^{\infty}$ and $x \neq y$, so that $d(x, y)=\infty$. Choose any $p \in X$. Let $\theta=x \hat{p} y>0$. Given any $r>0$, let $R=r \operatorname{cosec}(\theta / 4)$. Since $\tau\left(X_{C}\right)=\tau\left(X_{C}, p\right)$, by applying the Angle Comparison Theorem (Proposition 3.4.4), we can find neighbourhoods $U$ of $x$ and $V$ of $y$ such that if $u \in U$ and $v \in V$, then $d(p, u) \geq R$, $d(p, v) \geq R, x \hat{p} v \leq \theta / 4$ and $y \hat{p} v \leq \theta / 4$. Thus $u \hat{p} v \geq \theta / 2$ and so, again by angle comparison, $d(u, v) \geq r$.

5.2. The Hausdorff topology. We have seen that $X_{C}$ is homeomorphic to a ball and hence metrisable. A metric on $X_{C}$ induces a Hausdorff distance on the set, $\mathscr{C}\left(X_{C}\right)$, of all closed subsets of $X_{C}$ and hence a topology on $\mathscr{C}\left(X_{C}\right)$. Since $X_{C}$ is compact, it's not hard to see that the topology on on $\mathscr{C}\left(X_{C}\right)$ is independent of the choice of metric on $X_{C}$. We call this topology the Hausdorff topology on $\mathscr{C}\left(X_{C}\right)$.

A more natural description of the Hausdorff topology is in terms of uniformities (see $[\mathbf{K}]$ ). Here we shall deal only with bases of uniformities. Given a set $Y$, write $\Delta=\Delta(Y) \subseteq Y \times Y$ for the diagonal $\{(x, x) \mid x \in Y\}$. Given a subset $W \subseteq Y \times Y$, write $W^{2}=\{(x, y) \in Y \times Y \mid(\exists z \in Y)((x, z) \in W,(z, y) \in$ $W)\}$. We say that a subset $W \subseteq Y \times Y$ is symmetric if $(x, y) \in W$ whenever $(y, x) \in W$. A collection $\mathscr{W}$ of symmetric subsets of $Y \times Y$ form a uniform basis for $Y$ if the following hold:

(1) $\Delta \subseteq W$ for all $W \in \mathscr{W}$.

(2) For all $W_{1}, W_{2} \in \mathscr{W}$, there is some $W_{3} \in \mathscr{W}$ with $W_{3} \subseteq W_{1} \cap W_{2}$.

(3) For all $W \in \mathscr{W}$, there is some $V \in \mathscr{W}$ with $V^{2} \subseteq W$.

Two such bases $\mathscr{W}_{1}$ and $\mathscr{W}_{2}$ are equivalent if for all $W_{1} \in \mathscr{W}_{1}$ there is some $W_{2} \in \mathscr{W}_{2}$ with $W_{2} \subseteq W_{1}$, and for all $W_{2}^{\prime} \in \mathscr{W}_{2}$ there is some $W_{1}^{\prime} \in \mathscr{W}_{1}$ with $W_{1}^{\prime} \subseteq W_{2}^{\prime}$. Thus, two bases give rise to the same uniformity if and only if they are equivalent. (For our purposes, we can define a uniformity as an equivalence class of bases.)

Given a subset $W \subseteq Y \times Y$ and a subset $A \subseteq Y$, write $W A=\{x \dot{\epsilon}$ $Y \mid(\exists y \in A)((x, y) \in W)\}$. Thus if $\Delta \subseteq W$, then $A \subseteq W A$.

A uniform basis $\mathscr{W}$ on $Y$ induces a topology on $Y$, where a neighbourhood of the point $x \in Y$ is given by $\mathscr{W}\{x\}=\{W\{x\} \mid W \in \mathscr{W}\}$. This topology depends only on the uniformity. It is hausdorff if and only if $\bigcap \mathscr{W}=\Delta$. 
Note that a metric $d$ on $Y$ induces a uniformity with basis $\{\{(x, y) \in$ $Y \times Y \mid d(x, y) \leq \epsilon\} \mid \epsilon>0\}$. This uniformity, in turn, induces the metric topology. If $Y$ is compact, then this is the unique uniformity of $Y$ inducing the metric topology.

Suppose that $\mathscr{W}$ is a uniform basis on $Y$. Write $\mathscr{C}(Y)$ for the set of subsets that are closed in the induced topology. Given $W \in \mathscr{W}$, write $P(W)=$ $\{(A, B) \in \mathscr{C}(Y) \times \mathscr{C}(Y) \mid A \subseteq W B, B \subseteq W A\}$, and set $P(\mathscr{W})=\{P(W) \mid W \in$ $\mathscr{W}\}$. One checks that $P(\mathscr{W})$ is a uniform basis on $\mathscr{C}(Y)$. If $(Y, \mathscr{W})$ is hausdorff (respectively metrisable) then $(\mathscr{C}(Y), P(\mathscr{W}))$ is hausdorff (metrisable). We refer to the topology induced on $\mathscr{C}(Y)$ by $P(\mathscr{W})$ as the Hausdorff topology.

Since $X_{C}$ is compact metrisable, it admits a unique uniformity, and so $\mathscr{C}\left(X_{C}\right)$ has a well-defined Hausdorff topology. In the next section shall show that geodesics vary continuously in this topology. We spend the rest of this section giving an explicit description of the uniformity on $X_{C}$.

Fix $p \in X$, and suppose that $A \subseteq X \cup X_{I}^{0}$. Given $\epsilon>0$, define $\Omega(p, A, \epsilon) \subseteq$ $X_{C} \times X_{C}$ as follows. The pair $(x, y)$ lies in $\Omega(p, A, \epsilon)$ if either there is some $a \in A$ with $d(a,[p, x]) \leq \epsilon$ and $d(a,[p, y]) \leq \epsilon$, or else if $x, y \in X \cup X_{I}^{0}$ and $d(x, y) \leq 2 \epsilon$.

Clearly $\Omega(p, A, \epsilon)$ is symmetric and if $B \subseteq A$ and $\delta \leq \epsilon$, then $\Omega(p, B, \delta) \subseteq$ $\Omega(p, A, \epsilon)$.

Lemma 5.2.1. For all $A \subseteq X \cup X_{I}^{0}$ and $\epsilon>0$, we have $\Omega(p, A, \epsilon)^{2} \subseteq$ $\Omega(p, A, 3 \epsilon)$.

Proof. Suppose $(x, y),(y, z) \in \Omega(p, A, \epsilon)$. There are three cases.

(1) There are points $a, b \in A, a_{0} \in[p, x], a_{1}, b_{1} \in[p, y]$ and $b_{0} \in[p, z]$ with $d\left(a, a_{i}\right) \leq \epsilon$ and $d\left(b, b_{i}\right) \leq \epsilon$ for $i=0,1$. Without loss of generality, we have $d\left(p, b_{1}\right) \geq d\left(p, a_{1}\right)$. (Figure $\left.5 \mathrm{a}\right)$.

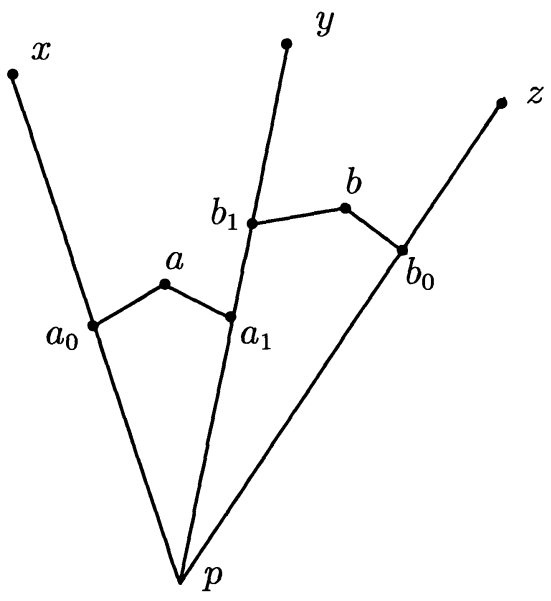

Figure 5a. 
Applying CAT(0) to $p b_{0} b_{1}$, we have $d\left(a_{1},[p, z]\right) \leq 3 \epsilon$. Thus $(x, z) \in$ $\Omega(p, A, 3 \epsilon)$.

(2) $d(y, z) \leq 2 \epsilon$ and there is some $a \in A$ with $d(a,[p, x]) \leq \epsilon$ and $d(a,[p, y]) \leq$ $\epsilon$. Applying $\operatorname{CAT}(0)$ to $p y z$, we find that $d(a,[p, z]) \leq 3 \epsilon$, and so $(x, z) \in \Omega(p, A, \epsilon)$.

(3) If $d(x, y) \leq 2 \epsilon$ and $d(y, z) \leq 2 \epsilon$, then $d(x, z) \leq 4 \epsilon$ and so $(x, z) \epsilon$ $\Omega(p, A, 2 \epsilon)$.

Given $r>0$, write $A(p, r)=X_{I}^{0} \cup(X \backslash \operatorname{int} N(p, r))$, and set $W(p, r, \epsilon)=$ $\Omega(p, A(p, r), \epsilon)$. Clearly $\Delta \subseteq W(p, r, \epsilon)$ for all $r>0$ and $\epsilon>0$. Let $\mathscr{W}=$ $\mathscr{W}_{p}=\{W(p, r, \epsilon) \mid r>0, \epsilon>0\}$. Applying Lemma 5.2.1, we see that $\mathscr{W}$ is a uniform base on $X_{C}$.

Lemma 5.2.2. The uniform base $\mathscr{W}_{p}$ induces the topology $\tau\left(X_{C}\right)$ on $X_{C}$.

Proof. We need to check that if $x \in X_{C}$, then $\mathscr{W}\{x\}$ gives a neighbourhood base for $x$ in $\tau\left(X_{C}\right)=\tau\left(X_{C}, p\right)$.

Case (1): $x \in X$.

If $\epsilon<d\left(X_{I}^{0},[x, p]\right)$ and $r>d(x, p)+\epsilon$, then $W(p, r, \epsilon)\{x\}=N(x, \epsilon)$.

Case (2): $x \in X_{I}^{0}$.

Clearly $C(p, x, \epsilon) \subseteq W(p, r, \epsilon)\{x\}$ for all $r>0$ and $\epsilon>0$. Now, $[p, x] \cap X_{I}^{0}=$ $\{x\}$. Given any $\epsilon \in(0, d(p, x))$, let $y \in[p, x]$ be the point with $d(x, y)=\epsilon / 3$. Let $\delta=\delta(\epsilon)=\frac{1}{2} d\left(X_{I}^{0},[p, y]\right)>0$, so $\delta \leq \epsilon / 6$. Now, suppose $r>d(p, x)+\delta$. If $z \in W(p, r, \delta)\{x\}$, then either $d(z, x) \leq 2 \delta \leq \epsilon$, and so $z \in C(p, x, \epsilon)$, or else there is some $a \in A(p, r)$ with $d(a,[p, x]) \leq \delta$ and $d(a,[p, z]) \leq \delta \leq \epsilon / 3$. Since $r>d(p, x)+\delta$, we must have $a \in X_{I}^{0}$, and so $d(x, a) \leq \delta+\epsilon / 3 \leq 2 \delta / 3$. It follows that $d(x,[p, z]) \leq 2 \epsilon / 3+\epsilon / 3=\epsilon$, and again we have $z \in C(p, x, \epsilon)$. We have shown that $W(p, r, \delta)\{x\} \subseteq C(p, x, \epsilon)$.

Case (3): $x \in X_{I}^{\infty}$.

Given $r>0$, take $y \in[p, x]$ with $d(p, y)=r$. Then $C(p, r, \epsilon)\{x\} \subseteq$ $W(p, r, \epsilon)\{x\}$ for all $\epsilon>0$.

Conversely, suppose $y \in[p, x]$. Let $r=d(p, y)$, and let $\delta=\delta(r)=$ $\frac{1}{2} d\left(X_{I}^{0},[p, y]\right)>0$. Suppose $\epsilon \in(0, \delta)$, and $z \in W(p, r, \epsilon)\{x\}$. Then, there is some $a \in A(p, r)$ with $d(a,[p, y]) \leq \epsilon \leq \delta$ and $d(a,[p, z]) \leq \epsilon$. If $a \in X_{I}^{0}$, then $d(a,[p, y])>\delta$ and so $d(a,[x, y]) \leq \epsilon \leq 2 \epsilon$. If $a \notin X_{I}^{0}$, then $d(p, a) \geq r$, and so again, $d(a,[x, y]) \leq 2 \epsilon$. Applying $\operatorname{CAT}(0)$, we find that $d(y,[p, z]) \leq 3 \epsilon$ and so $z \in C(p, y, 3 \epsilon)$. Thus $W(p, r, \epsilon)\{x\} \subseteq C(p, y, 3 \epsilon)$.

It follows that the uniform base $\mathscr{W}_{p}$ defines the unique uniformity on $X_{C}$ inducing the topology $\tau\left(X_{C}\right)$. In particular, $\mathscr{W}_{p}$ and $\mathscr{W}_{q}$ are equivalent for all $p, q \in X$. 
5.3. Continuity of geodesics. By Lemma 4.1.4, any pair of points $(x, y) \in$ $\left(X_{C} \times X_{C}\right) \backslash\left(X_{I}^{\infty} \times X_{I}^{\infty}\right)$ may be joined by a unique geodesic $[x, y]$.

Lemma 5.3.1. Each geodesic $[x, y]$ is closed $X_{C}$.

Proof. We can assume $x \neq y$. Choose $p \in[x, y] \backslash\{x, y\}$. If $z_{n} \in[x, y]$ is any sequence, it is easily seen that some subsequence converges in $\tau\left(X_{C}, p\right)$ to a limit in $[x, y]$.

We give $\mathscr{C}\left(X_{C}\right)$ the Hausdorff topology as described in Section 5.2. We give $X_{C} \times X_{C}$ the product topology $\tau\left(X_{C}\right) \times \tau\left(X_{C}\right)$.

Proposition 5.3.2. The map $[(x, y) \mapsto[x, y]]:\left(X_{C} \times X_{C}\right) \backslash\left(X_{I}^{\infty} \times X_{I}^{\infty}\right) \longrightarrow$ $\mathscr{C}\left(X_{C}\right)$ is continuous.

Proof. We distinguish six cases.

Case (1): $x, y \in X$.

This follows from Proposition 3.4.2.

Case (2): $x, y \in X_{I}^{0}$ and $x \neq y$.

Fix some $p \in[x, y] \cap X$. Suppose $r>0$ and $\epsilon>0$. Let $U, V$ be the neighbourhoods of $x, y$ respectively, given by Lemma 5.1.1, so that if $u \in U$ and $v \in V$, then we can find $u^{\prime}, v^{\prime} \in[u, v]$ with $d\left(x, u^{\prime}\right) \leq \epsilon / 2, d\left(y, v^{\prime}\right) \leq \epsilon / 2$ and $u^{\prime} \in\left[u, v^{\prime}\right]$. From the convexity of the distance function (Proposition 3.4.6), we have that $\left[u^{\prime}, v^{\prime}\right] \subseteq N([x, y], \epsilon / 2) \subseteq W(p, r, \epsilon)[x, y]$ and $[x, y] \subseteq$ $N\left(\left[u^{\prime}, v^{\prime}\right], \epsilon / 2\right) \subseteq W(p, r, \epsilon)[u, v]$. (Figure 5b.) Suppose $z \in\left[u, u^{\prime}\right]$. Again, by convexity, we have $d\left(u^{\prime},[p, z]\right) \leq \epsilon / 2$, and so $d(x,[p, z]) \leq \epsilon$. Thus $z \in$ $C(p, x, \epsilon) \subseteq W(p, r, \epsilon)\{x\}$. Therefore, $\left[u, u^{\prime}\right] \subseteq W(p, r, \epsilon)\{x\}$. Similarly, $\left[v, v^{\prime}\right] \subseteq W(p, r, \epsilon)\{y\}$. We have shown that

$$
[u, v] \subseteq W(p, r, \epsilon)[x, y]
$$

and

$$
[x, y] \subseteq W(p, r, \epsilon)[u, v]
$$

In other words, $[u, v] \in P(W(p, r, \epsilon))\{[x, y]\}$. Now, the sets

$$
P(W(p, r, \epsilon))\{[x, y]\}
$$

as $\epsilon \rightarrow 0$ and $r \rightarrow \infty$ form a neighbourhood base for $[x, y]$ in the Hausdorff topology on $\mathscr{C}\left(X_{C}\right)$. This deals with Case (2).

Case (3): $x=y \in X_{I}^{0}$.

Choose any $p \in X$, and suppose $\epsilon>0$ and $r>0$. By Lemma 4.3.2, there is some $\delta_{0}>0$ such that if $a, z \in X_{C}$ with $d(x, a) \leq \delta_{0}$ and $z \hat{a} p \geq \pi / 3$, 
then $d(x,[p, z]) \leq \epsilon$. Let $\delta=\min \left(\delta_{0}, \epsilon / 3\right)$. Suppose $u, v \in C(p, x, \delta)$, and $(u, v) \notin X_{I}^{\infty} \times X_{I}^{\infty}$. We claim that $[u, v] \subseteq C(p, x, \epsilon)$.

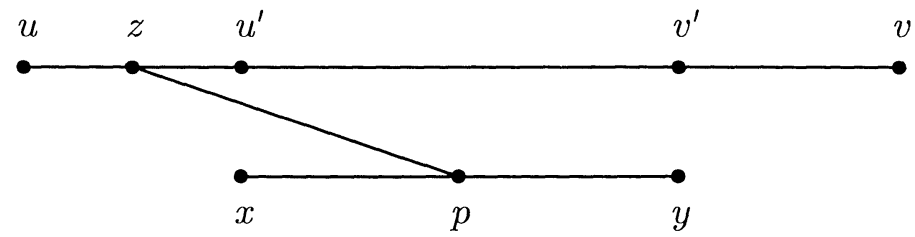

Figure 5b.

To see this, choose $a \in[p, u]$ and $b \in[p, v]$ with $d(x, a) \leq \delta$ and $d(x, b) \leq \delta$, and suppose $z \in[u, v]$. (Figure 5c.) If $d(a, z) \leq 2 \delta$, then $d(x, z) \leq \delta+2 \delta \leq \epsilon$, and so $z \in N(x, \epsilon) \subseteq C(p, x, \epsilon)$. Similarly if $d(b, z) \leq 2 \delta$. Thus, we can suppose that $d(a, z) \geq 2 \delta$ and $d(b, z) \geq 2 \delta$, and so, by the Angle Comparison Theorem, we have that $a \hat{z} b \leq \pi / 3$. Thus, without loss of generality, we can suppose that $u \hat{z} a \geq \frac{1}{2}(\pi-\pi / 3)=\pi / 3$. Thus, again by angle comparison, $u \hat{a} z \leq \pi-\pi / 3=2 \pi / 3$, and so $z \hat{a} p \geq \pi / 3$. It follows that $d(a,[p, z]) \leq \epsilon$, and so $z \in C(p, x, \epsilon)$. This proves the claim that $[u, v] \subseteq C(p, x, \epsilon)$.

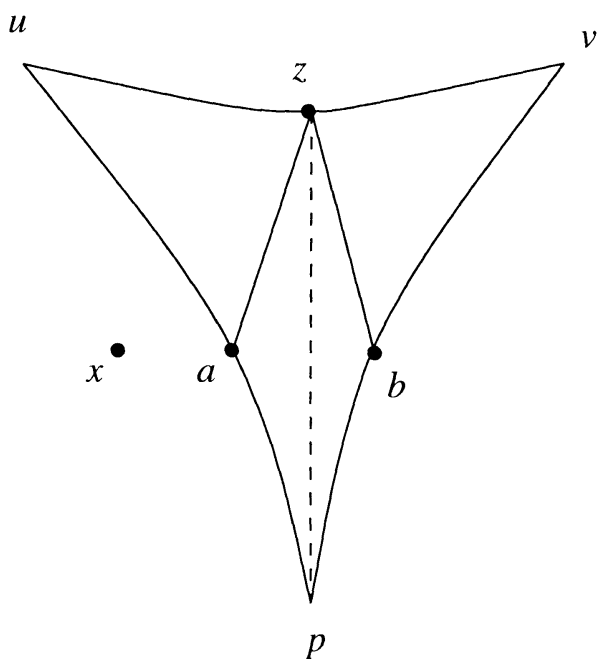

Figure 5c.

Now, for all $r>0$, we have $C(p, x, \epsilon) \subseteq W(p, r, \epsilon)\{x\}$. Since $W(p, r, \epsilon)$ is symmetric, we have $x \in W(p, r, \epsilon)[u, v]$, and so $[u, v] \in P(W(p, r, \epsilon))\{\{x\}\}$. As $\epsilon \rightarrow 0$ and $r \rightarrow \infty$, the sets $P(W(p, r, \epsilon))\{\{x\}\}$ form a neighbourhood base for $\{x\}=[x, x]$ in the Hausdorff topology on $\mathscr{C}\left(X_{C}\right)$.

Case (4): $x \in X$ and $y \in X_{I}^{0}$.

This is similar to Case (2). 
Case (5): $x \in X_{I}^{0}$ and $y \in X_{I}^{\infty}$.

Fix $p \in[x, y] \backslash\{x, y\}$, and suppose $\epsilon>0$ and $r>0$. Choose $z \in[p, y]$ with $d(p, z) \geq r$. By Lemma 5.1.3, we can find neighbourhoods $U, V$ about $x, y$ respectively, such that if $u \in U, v \in V$ and $(u, v) \notin X_{I}^{\infty} \times X_{I}^{\infty}$, then there exist $u^{\prime}, v^{\prime} \in[u, v]$ with $d\left(x, u^{\prime}\right) \leq \epsilon / 2, d\left(y, v^{\prime}\right) \leq \epsilon / 2$ and $u^{\prime} \in\left[u, v^{\prime}\right]$. Arguing as in Case (2), we see that $\left[u^{\prime}, v^{\prime}\right] \subseteq N([x, y], \epsilon / 2),[x, z] \subseteq N([u, v], \epsilon / 2)$, $\left[u, u^{\prime}\right] \subseteq C(p, x, \epsilon),\left[v, v^{\prime}\right] \subseteq C(p, z, \epsilon)$ and $[z, y] \subseteq C\left(p, v^{\prime}, \epsilon / 2\right)$. Now $x, z \in$ $A(p, r)$ and so $[u, v] \in P(W(p, r, \epsilon))\{[x, y]\}$.

Case (6): $x \in X$ and $y \in X_{I}^{\infty}$.

This is similar to case (5).

\section{Visibility.}

In this Chapter, we assume that $X$ satisfies properties (A), (B) and (C), where $(\mathrm{C})$ is the statement:

(C) There exist $p_{0} \in X$, and $L_{0}, R_{0}>0$ such that if $x \in X$ with $d\left(p_{0}, x\right) \geq$ $R_{0}$, then $\kappa(x) \leq-1 / L_{0}^{2} d\left(p_{0}, x\right)^{2}$.

It follows immediately that if we fix any $L \in\left(0, L_{0}\right)$, then for all $p \in X$, there is some $R=R(p)$ such that if $d(p, x) \geq R$, then $\kappa(x) \leq-1 / L^{2} d(p, x)$. We aim to show that, with these hypotheses, $X$ is a visibility manifold, and that geodesics vary continuously on $X_{C} \times X_{C}$.

6.1. Convergence of asymptotic geodesics. Suppose $y \in X_{I}^{\infty}$, and $h$ : $X \cup X_{I}^{0} \longrightarrow \mathbb{R}$ is a horofunction about $y$. (Section 4.2.) Suppose $b_{0}, b_{1} \in$ $X \cup X_{I}^{0}$ with $h\left(b_{0}\right)=h\left(b_{1}\right)$. Let $\beta_{i}:[0, \infty) \rightarrow X \cup X_{I}^{0}$ be the geodesic ray $\left[b_{i}, y\right]$. Thus $h\left(\beta_{0}(t)\right)=h\left(\beta_{1}(t)\right)=h\left(b_{0}\right)+t$ for all $t \in[0, \infty)$.

Lemma 6.1.1. $d\left(\beta_{0}(t), \beta_{1}(t)\right) \rightarrow 0$ as $t \rightarrow \infty$.

In fact, we show that $d\left(\beta_{0}(t), \beta_{1}(t)\right) \leq A(t+\lambda)^{-\mu}$ where $\mu>0$ is fixed, and $A, \lambda \geq 0$ depend on $b_{0}$ and $b_{1}$.

Proof. We can assume that $b_{0}, b_{1} \in X$. Join $b_{0}$ to $b_{1}$ by a smooth path $\gamma:[0,1] \longrightarrow X$. Let $t_{0}=\max \{h(\gamma(u)) \mid u \in[0,1]\}$. Let $B$ be the horoball $X \cap h^{-1}\left(\left[t_{0}, \infty\right)\right)$, and let $S$ be the bounding horosphere $X \cap h^{-1}\left(t_{0}\right)$. Let $\rho: X \backslash \operatorname{int} B \longrightarrow S$ be the nearest-point retraction. Now, the path $h \circ \gamma:$ $[0,1] \longrightarrow S$ joins $\beta\left(t_{0}\right)$ to $\beta\left(t_{1}\right)$, and, by Lemma 4.2 .2 , is $C^{2}$. Thus, without loss of generality, we can assume that $b_{0}, b_{1} \in S=h^{-1}(0)$, and that $b_{0}$ and $b_{1}$ can be joined by a $C^{2}$ path $\gamma:[0,1] \longrightarrow S$.

Now, for each $u \in[0,1]$, let $\beta_{u}:[0, \infty) \rightarrow X$ be the geodesic ray based at $\gamma(u)$ tending to $y$. Define $\beta:[0, \infty) \times[0,1] \longrightarrow X$ by $\beta(t, u)=\beta_{u}(t)$. By Lemma $4.1 .5, \beta$ is $C^{2}$. Note that $h(\beta(t, u))=t$ for all $(t, u) \in[0, \infty) \times[0,1]$. 
Also $\frac{\partial \beta}{\partial u}(t, u)=\operatorname{grad} \beta(h(t, u))$. Thus $\left\langle\frac{\partial \beta}{\partial t}(t, u), \frac{\partial \beta}{\partial u}(t, u)\right\rangle=0$ for all $(t, u)$. In other words $\beta$ is a normalised ruled map in the sense of Section 3.1 (except that it is only $C^{2}$ and not smooth, though this is more than enough). For a fixed $u$, the map $\left[t \mapsto \frac{\partial \beta}{\partial u}(t, u)\right]$ is a Jacobi field along $\beta_{u}$. Thus the map $[t \mapsto J(t, u)]$ is convex, where $J(t, u)=\left|\frac{\partial \beta}{\partial u}(t, u)\right|$. Given $t \in[0, \infty)$ write $\beta^{t}:[0,1] \longrightarrow X$ for the $C^{2}$ transversal path $[u \mapsto \beta(t, u)]$. Thus length $\beta^{t}=$ $\int_{0}^{1} J(t, u) d u$. Now, for all $u_{1}, u_{2} \in[0,1]$ the function $d\left(\beta\left(t, u_{1}\right), \beta\left(t, u_{2}\right)\right)$ is monotonically non-increasing in $t$. Thus, for any fixed subinterval $I \subseteq$ $[0,1]$, the rectifiable lengths of the paths $\beta^{t} \mid I$ are non-increasing in $t$. Now, length $\left(\beta^{t} \mid I\right)=\int_{I} J(t, u) d u$. We deduce that for all $u \in[0,1]$ the map $[t \mapsto$ $J(t, u)]$ is non-increasing.

Now choose $p \in X$, and let $R=R(p)$. Thus, if $d(p, x) \geq R$, then we have $\kappa(x) \leq-1 / L^{2} d(p, x)^{2}$. Let $\lambda=\max \{d(p, \gamma(u)) \mid u \in[0,1]\}$. Thus $t-\lambda \leq d(p, \beta(t, u)) \leq t+\lambda$. Without loss of generality, we can assume that $d(p, \beta(t, u)) \geq R$ for all $(t, u)$, and so $\kappa(\beta(t, u)) \leq-1 / L^{2}(t+\lambda)^{2}$.

From the formula in Section 4.2, we find that $J(t, u) \leq J(0, u)\left(1+\frac{t}{\lambda}\right)^{-\mu}$ where $\mu=\left(\sqrt{1+4 L^{2}}\right)-1>0$. Thus

$$
\begin{aligned}
d\left(\beta_{0}(t), \beta_{1}(t)\right) & \leq \text { length } \beta^{t}=\int_{0}^{1} J(t, u) d u \\
& \leq\left(1+\frac{t}{\lambda}\right)^{-\mu} \text { length } \gamma=A(t+\lambda)^{-\mu},
\end{aligned}
$$

where $A=\lambda^{\mu}$ length $\gamma$. In particular $d\left(\beta_{0}(t), \beta_{1}(t)\right) \rightarrow 0$ as $t \rightarrow \infty$.

6.2. Bi-infinite geodesics. A bi-infinite geodesic is a geodesic $\beta: \mathbb{R} \longrightarrow X$ parameterised by arc-length. We say that $\beta$ joins $x \in X_{I}^{\infty}$ to $y \in X_{I}^{\infty}$ if $\beta(-t) \rightarrow x$ and $\beta(t) \rightarrow y$ as $t \rightarrow \infty$. Clearly the points $x$ and $y$ are determined by $\beta$. We refer to them as the "endpoints" of $\beta$. Since $d(\beta(-t), \beta(t))=2|t|$, the rays $[t \mapsto \beta(-t)]$ and $[t \mapsto \beta(t)]$ for $t \geq 0$ are not asymptotic. Thus the endpoints of $\beta$ must be distinct. Moreover, the endpoints determine $\beta$ up to reparameterisation:

Lemma 6.2.1. Suppose that the bi-infinite geodesics $\alpha, \beta: \mathbb{R} \rightarrow X$ have the same endpoints. Then, there is some $t_{0} \in \mathbb{R}$ such that $\beta(t)=\alpha\left(t+t_{0}\right)$.

Proof. Let $y \in X_{I}^{\infty}$ be the common endpoint so that $\alpha \rightarrow y$ and $\beta \rightarrow$ $y$ as $t \rightarrow \infty$. Let $h$ be a horofunction about $y$. There is some $t_{0} \in \mathbb{R}$ such that $h\left(\alpha\left(t+t_{0}\right)\right)=h(\beta(t))$ for all $t \in \mathbb{R}$. By Lemma 6.1.1, we have $d\left(\alpha\left(t+t_{0}\right), \beta(t)\right) \rightarrow 0$ as $t \rightarrow \infty$. Also $d\left(\alpha\left(t+t_{0}\right), \beta(t)\right)$ is bounded as $t \rightarrow-\infty$. By Proposition 3.5.6, the map $\left[t \mapsto d\left(\alpha\left(t+t_{0}\right), \beta(t)\right)\right]$ is convex, and thus identically zero. 
We next want to establish the existence of a bi-infinite geodesic joining any pair of distinct points of $X_{I}^{\infty}$.

Lemma 6.2.2. Suppose $p \in X$. Then, for all $\theta>0$, there exists $r>0$ such that $x, y \in X \cup X_{I}^{\infty}$, then either $d(p,[x, y]) \leq r$ or else $x \hat{a} y \leq \theta$.

Proof. Let $R=R(p)$ and $L>0$ be the constants defined at the start of this chapter. Let $r=R \max \left(1, e^{2 \pi L^{2} / \theta}\right)$. Suppose, for contradiction, that $d(p,[x, y]) \geq r$, and $x \hat{p} y \geq \theta$. We form a ruled surface $T$ by joining $p$ to each point $w \in[x, y]$ with a geodesic $[p, w]$ (c.f. Lemma 4.3.2). Thus $T$ is a non-positively curved 3-gon with vertices $p, x$ and $y$. By Gauss-Bonnet, we have $-\int_{T} \kappa(z) d \omega(z) \leq \pi$ where $d \omega$ is the area element of $T$. As in Lemma 4.3.2, we obtain the contradiction:

$$
\begin{aligned}
\pi & \geq-\int_{T} \kappa(z) d \omega(z) \geq \int_{R}^{r} \frac{\theta t}{L^{2} t^{2}} d t \\
& =\frac{\theta}{L^{2}} \log (r / R) \geq 2 \pi .
\end{aligned}
$$

Proposition 6.2.3. If $x, y \in X_{I}^{\infty}$, and $x \neq y$, then there is a bi-infinite geodesic joining $x$ to $y$.

Proof. Fix any $p \in X$. Thus $x \hat{p} y>0$. Choose sequences $x_{n} \in[p, x] \cap X$ and $y_{n} \in[p, y] \cap X$ with $x_{n} \rightarrow x$ and $y_{n} \rightarrow y$. By Lemma 6.2.2, we can find points $z_{n} \in\left[x_{n}, y_{n}\right]$ with $d\left(p, z_{n}\right)$ bounded. Since $\left(X_{C}, \tau\left(X_{C}\right)\right)$ is compact metrisable, we can assume that $z_{n}$ converges to a point $z \in X_{C}$. By the lower-semicontinuity of the distance function (Proposition 5.1.4), we see that $d(p, z)<\infty$ and so $z \in X \cup X_{I}^{0}$. Thus, by Lemma 4.1.4, we can construct the geodesics $[z, x]$ and $[z, y]$.

Now choose any $a \in[z, x] \backslash\{z, x\}$ and $b \in[z, y] \backslash\{z, y\}$. We claim that $d(a, z)+d(z, b)=d(a, b)$. By Proposition 5.3.2, the geodesic $\left[z_{n}, x_{n}\right]$ tends to $[z, x]$ in the Hausdorff topology. Since the metric topology on $X$ agrees with that induced by $\tau\left(X_{C}\right)$, we have, in particular, that $d\left(a,\left[x_{n}, z_{n}\right]\right) \rightarrow 0$. Similarly $d\left(b,\left[y_{n}, z_{n}\right]\right) \rightarrow 0$. Thus we can find $a_{n} \in\left[x_{n}, z_{n}\right]$ and $b_{n} \in\left[y_{n}, z_{n}\right]$ with $d\left(a, a_{n}\right) \rightarrow 0$ and $d\left(b, b_{n}\right) \rightarrow 0$. Now $d\left(a_{n}, z_{n}\right)+d\left(z_{n}, b_{n}\right)=d\left(a_{n}, b_{n}\right)$ and so the claim follows. Thus, since $[a, b]$ is the unique geodesic from $a$ to $b$, we have that $z \in[a, b]$. It follows that $z \in X$, and $[x, z] \cup[z, y]$ gives a bi-infinite geodesic joining $x$ to $y$.

If $x, y \in X_{I}^{\infty}$ and $x \neq y$, we write $[x, y]=\{x, y\} \cup$ image $\beta$, where $\beta$ is the unique (up to parameterisation) geodesic joining $x$ to $y$. It is easily seen that $[x, y]$ is closed in $\left(X_{C}, \tau\left(X_{C}\right)\right)$. Note that $[x, y]=[y, x]$. We write $[x, x]=\{x\}$. 


\subsection{Continuity of geodesics.}

Lemma 6.3.1. Suppose $p \in X$, and $\kappa(p)<0$. Then for all $\epsilon>0$, there exists $\delta>0$ such that if $x, y \in X_{C} \backslash\{p\}$ with $x \hat{p} y \geq \pi-\delta$, then $d(p,[x, y]) \leq \epsilon$.

Proof. By continuity of $\kappa$, we have constants $h>0$ and $k>0$ such that $N(p, h) \subseteq X$ and $\kappa(z) \leq-k$ for all $z \in N(p, h)$. Given $\epsilon \in(0, h)$, let $\delta=$ $\min \left(\pi / 2, k \pi h^{2} / 4\right)$. Suppose that $x, y \in X_{C} \backslash\{p\}$ are distinct with $d(p,[x, y]) \geq$ $\epsilon$. Let $\theta=x \hat{p} y$. We claim that $\theta \leq \pi-\delta$. We can suppose that $\theta \geq \pi / 2$. For the moment, assume that $x, y \in X \cup X_{I}^{0}$. We form a ruled surface by joining each $w \in[x, y]$ to $p$ by the geodesic $[p, w]$ (c.f. Lemma 4.3.2). Integrating the curvature, we find that

$$
\pi-\theta \geq \int_{0}^{h} k\left(\frac{\pi}{2} t\right) d t=k \pi h^{2} / 4 \geq \delta
$$

Thus $\theta \leq \pi-\delta$ as required.

We can deal with the general case by taking the sequences $x_{n}, y_{n} \in$ $[x, y] \cap X$ with $x_{n} \rightarrow x$ and $y_{n} \rightarrow y$, and noting that $\overrightarrow{p x_{n}} \rightarrow \overrightarrow{p \vec{x}}$ and $\overrightarrow{p y_{n}} \rightarrow \overrightarrow{p y}$.

We give $X_{C} \times X_{C}$ the product topology, and give $\mathscr{C}\left(X_{C}\right)$ the Hausdorff topology.

Proposition 6.3.2. The map $[(x, y) \mapsto[x, y]]: X_{C} \times X_{C} \longrightarrow \mathscr{C}\left(X_{C}\right)$ is continuous.

Proof. Note that Lemmas 5.1.1 and 5.1.3 generalise easily to the case where $(u, v) \in X_{I}^{\infty} \times X_{I}^{\infty}$, with essentially the same proofs. Thus the argument of Proposition 5.3.2 works to show that the map $[(x, y) \mapsto[x, y]]$ extended to all of $X_{C} \times X_{C}$ is continuous at each point $(x, y) \in\left(X_{C} \times X_{C}\right) \backslash\left(X_{I}^{\infty} \times X_{I}^{\infty}\right)$. It thus remains to show that it is continuous at each point $(x, y) \in X_{I}^{\infty} \times X_{I}^{\infty}$. There are two cases.

Case (1): $x \neq y$.

Fix some $p \in[x, y] \cap X$ with $\kappa(p)<0$. Suppose $\epsilon>0$ and $r>0$. Let $\delta>0$ be the constant given by Lemma 6.3.1, and set $\eta=\min (\epsilon, r \sin (\delta / 4))$. Choose points $a \in[p, x]$ and $b \in[p, y]$, with $d(p, a)=d(p, b)=r+2 \epsilon$. Let $U=C(p, a, \eta)$ and $V=C(p, b, \eta)$. If $u \in U$ and $v \in V$, then by the Angle Comparison Theorem (Proposition 3.4.4), we find that $x \hat{p} u \leq \delta / 2$ and $y \hat{p} v \leq \delta / 2$. Thus $u \hat{p} v \geq \pi-\delta$ and so $d(p,[u, v]) \leq \epsilon$. Thus, there is some $q \in[u, v]$ with $d(p, q) \leq \epsilon$. If $u \in X_{I}^{\infty}$, then $[p, u]$ and $[q, u]$ are asymptotic, and so, since $d(a,[p, u]) \leq \eta \leq \epsilon$, we can find $u^{\prime} \in[q, u]$ with $d\left(a, u^{\prime}\right) \leq 2 \epsilon$. If $u \in X \cup X_{I}^{0}$, we can apply The Angle Comparison Theorem to 
find such a $u^{\prime}$. Similarly, we can find $v^{\prime} \in[q, v]$ with $d\left(b, v^{\prime}\right) \leq 2 \epsilon$. Note that $d\left(p, u^{\prime}\right) \geq r$ and $d\left(p, v^{\prime}\right) \geq r$. By convexity of the distance function, we have $\left[u^{\prime}, v^{\prime}\right] \subseteq N([a, b], 2 \epsilon)$ and $[a, b] \subseteq N\left(\left[u^{\prime}, v^{\prime}\right], 2 \epsilon\right)$. Also $[x, a] \subseteq C\left(p, u^{\prime}, 2 \epsilon\right)$ and $[y, b] \subseteq C\left(p, v^{\prime}, 2 \epsilon\right)$. If $z \in\left[u, u^{\prime}\right]$, then by angle comparison, applied to $z p q$, we see that $d\left(u^{\prime},[p, z]\right) \leq 2 \epsilon$, and so $d(a,[p, z]) \leq 4 \epsilon$. This shows that $\left[u, u^{\prime}\right] \subseteq C(p, a, 4 \epsilon)$. Similarly, $\left[v, v^{\prime}\right] \subseteq C(p, b, 4 \epsilon)$. Since $a, b, u^{\prime}, v^{\prime} \in$ $A(p, r)$, we have that $[x, y] \subseteq W(p, r, 4 \epsilon)[u, v]$ and $[u, v] \subseteq W(p, r, 4 \epsilon)[u, v]$. In other words, $[u, v] \in P(W(p, r, 4 \epsilon))\{[x, y]\}$. As $\epsilon \rightarrow 0$ and $r \rightarrow \infty$, the sets $P(W(p, r, 4 \epsilon))\{[x, y]\}$ form a neighbourhood base for $[x, y]$ in the Hausdorff topology on $\mathscr{C}\left(X_{C}\right)$.

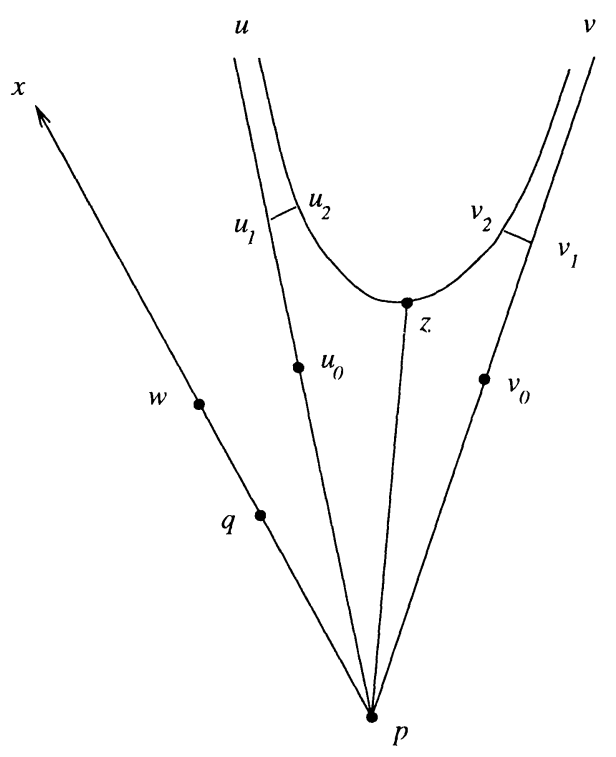

Figure 6.

Case (2): $x=y$.

Choose any point $p \in X$. Suppose $p \in X$. Suppose $r_{0}>0$ and $\epsilon>0$. Let $q \in[p, x]$ be the point with $d(p, q)=r_{0}$. By the continuity of the logarithm map (Section 4.5), there is some $\theta>0$ such that if $q^{\prime} \in X \cup X_{I}^{0}$ with $d\left(p, q^{\prime}\right)=r_{0}$ and $q \hat{p} q^{\prime} \leq 2 \theta$, then $q^{\prime} \in N(q, \epsilon)$. Thus if $z \in X_{C}$ with $d(p, z) \geq r_{0}$ and $x \hat{p} z \leq 2 \theta$, then $z \in C(p, q, \epsilon)$.

Given $\theta>0$, and $p \in X$, let $r>0$ by the constant given by Lemma 6.2.2. Choose any $\eta>0$ and let $R=\max \left(r_{0}+4 \eta, r+5 \eta, \eta \operatorname{cosec} \theta\right)$. Let $w \in[p, y]$ be the point with $d(p, w)=r$. Thus, by angle comparison, if $u \in C(p, w, \eta)$ then $y \hat{p} u \leq \theta$.

Now suppose that $u, v \in C(p, w, \eta)$. Choose $u_{0} \in[p, u]$ and $v_{0} \in[p, v]$ with $d\left(w, u_{0}\right) \leq \eta$ and $d\left(w, v_{0}\right) \leq \eta$. Suppose $z \in[u, v] \backslash\{u, v\}$. If $u \in X_{I}^{\infty}$, 
then $\left[u_{0}, u\right]$ and $[z, u]$ are asymptotic, and so we can find $u_{1} \in\left[u_{0}, u\right]$ and $u_{2} \in[z, u]$ with $d\left(u_{1}, u_{2}\right) \leq \eta$ (Lemma 6.1.1). If $u \in X_{I}^{0}$, take $u_{1}=u_{2}=u$. Similarly, we find $v_{1} \in\left[v_{0}, v\right]$ and $v_{2} \in[z, v]$ with $d\left(v_{1}, v_{2}\right) \leq \eta$. (Figure 6.) Thus

$$
\begin{aligned}
2 d(p, z) & \geq d\left(p, u_{1}\right)+d\left(p, v_{1}\right)-d\left(z, u_{1}\right)-d\left(z, v_{1}\right) \\
& \geq d\left(p, u_{0}\right)+d\left(p, v_{0}\right)+\left(d\left(u_{0}, u_{1}\right)+d\left(v_{0}, v_{1}\right)-d\left(u_{2}, v_{2}\right)\right)-2 \eta \\
& \geq 2 d(p, w)-8 \eta
\end{aligned}
$$

and so $d(p, z) \geq d(p, w)-4 \eta \geq \max \left(r_{0}, r+\eta\right)$. Since $z$ is arbitrary, we see that $d(p,[u, v]) \geq r+\eta$. Given this, we see in particular that $d\left(p,\left[z, u_{2}\right]\right) \geq r+\eta$ and so $d\left(p,\left[z, u_{1}\right]\right) \geq r$. Thus $z \hat{p} u_{1}=z \hat{p} u \leq \theta$. Since also $x \hat{p} u \leq \theta$ we have $x \hat{p} z \leq 2 \theta$. Since $d(p, z) \geq r$, it follows that $z \in C(p, q, \epsilon)$.

We have shown that if $u, v \in C(p, w, \eta)$, then $[u, v] \subseteq C(p, q, \epsilon)$. We deduce that $[u, v] \in P\left(W\left(p, r_{0}, \epsilon\right)\right)\{\{x\}\}$. As $r_{0} \rightarrow \infty$ and $\epsilon \rightarrow 0$, these sets form a neighbourhood base for $\{x\}=[x, x]$ in the Hausdorff topology on $\mathscr{C}\left(X_{C}\right)$.

\section{References}

[AbS] U. Abresch and V. Schroeder, Graph manifolds, ends of negatively curved spaces and the hyperbolic 120-cell space, J. Diff. Geom., 35 (1992), 299-336.

[Ah] L.V. Ahlfors, Curvature properties of Teichmüller space, J. d'Anal. Math., 9 (1961), 161-176.

[Alek] A.D. Aleksandrov, Ruled surfaces in metric spaces, Vestnik Leningrad Univ. 12 (1957), 5-26 (Russian).

[AlexB] S.B. Alexander and R.L.Bishop, The Hadamard-Cartan theorem in locally convex metric spaces, L'Enseign. Math. 36 (1990), 309-320.

[An] M.T. Anderson, The Dirichlet problem at infinity for manifolds of negative curvature, J. Diff. Geom., 18 (1983), 701-721.

[BaGS] W. Ballmann, M. Gromov and V.Schroeder, Manifolds of non-positive curvature, Progress in Maths., 61, Birkhäuser (1985).

[BiO] R.L. Bishop and B. O'Neill, Manifolds of negative curvature, Trans. Amer. Math. Soc., 145 (1969), 1-49.

[Bo] B.H. Bowditch, Some results on the geometry of convex hulls in manifolds of pinched negative curvature, Comment. Math. Helv., 69 (1994), 49-81.

[BrH] M.R. Bridson and A. Haefliger, Non-positively curved metric spaces, in preparation.

[EO] P. Eberlein and B. O'Neill, Visibility manifolds, Pacific J. Math., 46 (1973), 45-110.

[GH] E. Ghys, P.de la Harpe (ed.), Sur les groupes hyperboliques d'après Mikhael Gromov, Progress in Maths., 83, Birkhäuser (1990).

[Ha] J. Hass, Bounded 3-manifolds admit negatively curved metrics with concave boundary, J. Diff. Geom., 40 (1994), 449-459. 
[HeI] E. Heintze and H.C. Im Hof, Geometry of horospheres, J. Diff. Geom., 12 (1977), 481-491.

[K] J.L. Kelley, General topology, Graduate Texts in Maths., 21, Springer-Verlag (reprint of Van Nostrand edition 1955).

[S] M. Spivak, A comprehensive introduction to differential geometry, Publish or Perish (1979).

[Tra] S. Trapani, On the determinant of the bundle of meromorphic differentials on the Deligne-Mumford compactification of the moduli space of Riemann surfaces, Math. Annalen, 293 (1992), 481-459.

[Tro] A. Tromba, On a natural algebraic affine connection on the space of almost complex structures and the curvature of Teichmüller space with respect to its Weil-Peterssen metric, Manuscripta Math., 56 (1986), 475-497.

[W1] S.A. Wolpert, Non-completeness of the Weil-Peterssen metric for Teichmüller space, Pacific J. Math., 61 (1975), 573-577.

[W2] — Geodesic length functions and the Neilsen problem, J. Diff. Geom., 25 (1987), 275-296.

Received July 30, 1993 and revised June 12, 1995. This article was prepared at the University of Melbourne, with the support of an A.R.C. Fellowship.

UNIVERSITY OF SOUTHAMPTON

HighField, Southampton SO17 1BJ

Great Britain

E-mail address: bhb@maths.soton.ac.uk

Current address:

UNIVERSITY OF MELBOURNE

PARKVILle, Victoria 3052

Australia 




\section{PACIFIC JOURNAL OF MATHEMATICS}

Volume $172 \quad$ No. $1 \quad$ January 1996

A class of incomplete non-positively curved manifolds

BRIAN BOWDITCH

The quasi-linearity problem for $C^{*}$-algebras

41

L. J. BUNCE and JOHN DAVID MAITLAND WRIGHT

Distortion of boundary sets under inner functions. II

Jose Luis Fernandez Perez, Domingo Pestana and José Rodríguez

Irreducible non-dense $A_{1}^{(1)}$-modules

VJACHESLAV M. FUTORNY

$M$-hyperbolic real subsets of complex spaces

101

Giuliana Gigante, Giuseppe Tomassini and Sergio Venturini

Values of Bernoulli polynomials

ANDREW GRANVILLE and ZHI-WEI SUN

The uniqueness of compact cores for 3-manifolds

LUKE HARRIS and PETER SCOTT

Estimation of the number of periodic orbits

BOJU JIANG

Factorization of $p$-completely bounded multilinear maps

Christian Le MERdy

Finitely generated cohomology Hopf algebras and torsion

JAMEs Peicheng LiN

The positive-dimensional fibres of the Prym map

JUAN-CARLOS NARANJO

Entropy of a skew product with a $Z^{2}$-action

KYEWON KOH PARK

Commuting co-commuting squares and finite-dimensional Kac algebras

TAKASHI SANO

Second order ordinary differential equations with fully nonlinear two-point boundary 255 conditions. I

H. BEVAN THOMPSON

Second order ordinary differential equations with fully nonlinear two-point boundary conditions. II

H. BEVAN THOMPSON

The flat part of non-flat orbifolds

FENG XU 